[Vicino Oriente XVI (2012), pp. 71-96]

\title{
UN ALTARE BRUCIAPROFUMI PUNICO DALLA “CASA DEL SACELLO DOMESTICO” A MOZIA
}

\author{
Federica Spagnoli - Sapienza Università di Roma ${ }^{1}$
}

Excavations at Motya carried on by «La Sapienza» University of Rome to the western slope of the Acropolis (Area D) have brought to light a small altar/incense burner which illustrates a well attested typology of domestic cult devices of Phoenician-Punic tradition. The context of the cult installation provides some interesting hints at its religious utilizations.

Keywords: altare bruciaprofumi; Mozia; punico; culto domestico; rituale funerario

\section{INTRODUZIONE}

Le campagne di scavo effettuate continuativamente negli anni 2002-2011 a Mozia dall’Università di Roma «La Sapienza» sotto la direzione del Professor Lorenzo Nigro, nell'area denominata Zona D, alle pendici occidentali dell'Acropoli (fig. 1), hanno portato alla luce un vasto edificio interpretato come una residenza aristocratica in uso nella seconda metà del V secolo a.C. (fig. 2), dato alle fiamme durante la distruzione dionigiana del 397/6 a.C. Proprio questa evenienza storico-archeologica ha fatto sì che gli oggetti e gli arredi in uso al momento del saccheggio siracusano siano rimasti conservati nello strato di crollo che si estendeva su tutte le rovine dell'edificio.

\section{IL CONTESTO DI RINVENIMENTO: ARCHITETTURA E MATERIALI}

La residenza aristocratica, denominata "Casa del sacello domestico" per via dell'installazione adibita al culto domestico rinvenuta al suo interno ${ }^{2}$, si articola attorno ad una corte rettangolare ed ha l'ingresso in un angolo, aperto su una piazza sulla quale si affacciavano altre abitazioni signorili ${ }^{3}$ e dalla quale parte una strada che discende dall'acropoli verso la città bassa e il Kothon ${ }^{4}$. Dall'ampio portale, segnato da una soglia in blocchi di calcarenite, sotto la quale era un deposito di fondazione costituito da un'olpetta in ceramica comune (fig. 3) $)^{5}$, si accedeva ad una sala d'ingresso con banchine e sedili in

1 Questo contributo è stato presentato come comunicazione al VI Congresso Internazionale di Archeologia Fenicio-Punica svoltosi a Lisbona dal 1 al 6 ottobre del 2005. Desidero ringraziare il Professor Lorenzo Nigro, Direttore della Missione Archeologica a Mozia, per avermi affidato lo studio di questo reperto e del suo contesto di rinvenimento.

2 Nigro 2004a, 200-202.

3 A sud della strada L.261, che borda il lato sud-orientale della "Casa del sacello domestico", è stato rinvenuto un secondo edificio, denominato Edificio D3 o "Casa del corno di conchiglia”, risalente al V secolo a.C. Nel vano centrale dei tre venuti alla luce dell'Edificio D3 è stata rinvenuta, infatti, una grande conchiglia marina deposta in una nicchia delimitata da piccole lastre disposte di taglio nell'angolo interno; si trattava di una Charonia Tritonis Nodifera finemente lavorata. La conchiglia era probabilmente parte di un deposito votivo (D.2205) che includeva anche una punta di freccia di bronzo, un piccolo skyphos a vernice nera, un kotyliskos inciso, una brocchetta a vernice nera contenente ossa di uccello e conchiglie, una coppetta e l'orlo di una lekythos. La ricchezza dei rinvenimenti effettuati all'interno dell'edificio ne attestano la natura di residenza di lusso (Nigro 2009, 552-553).

$4 \quad$ Nigro 2003, 89-93; Nigro 2004a, 143, fig. 3:1; Nigro 2007b, 53-54.

$5 \quad$ Nigro 2004a, 167, figg. 3:20-3:21, tavv. LXIII, LXXXVII. 
mattoni crudi intervallate da pilastri portanti e un fine pavimento in marna calcarea pressata e argilla (fig. 4). Già nella prima campagna di scavi era stato notato l'elevato status del proprietario della "Casa del sacello domestico", segnalato dal rinvenimento, proprio in questa sala, di alcuni vasi a figure rosse in frammenti, come due crateri, uno dei quali a campana di produzione siceliota attribuito al Pittore di Meleagro o ai ceramografi della sua cerchia, e quindi ascrivibile alla fine del V-inizi del IV secolo a.C. (fig. 5) ${ }^{6}$, e uno skyphos a vernice nera (fig. 6) ${ }^{7}$. Dalla sala d'ingresso si passava ad un vestibolo con funzioni di ambiente di distribuzione dal quale si accedeva rispettivamente ad ovest ad un magazzino nel quale sono state rinvenute numerose anfore puniche e a nord ad un piccolo ambiente (L.300) caratterizzato dalla presenza di alcune installazioni di carattere cultuale (fig. 7): un arredo fittile conformato a capitello eolico ${ }^{8}$, un'arula in terracotta ${ }^{9}$, un astragalo, una grappa in ferro e una moneta ${ }^{10}$ interpretabili come offerta votiva, una coppetta miniaturistica a vernice nera ${ }^{11}$, una coppetta in ceramica comune punica e un incensiere frammentario ${ }^{12}$; di fronte era collocato un apprestamento semicircolare in argilla con evidenti tracce di bruciato all'interno ${ }^{13}$. Su un piano rialzato rispetto al sacello era un bagno del quale si conservano, oltre alla pavimentazione, il rivestimento parietale in intonaco idraulico rosso, la vasca in argilla a sabot e due anfore puniche originariamente ad essa appoggiate (fig. $8)^{14}$. Sempre dallo stesso vestibolo si accedeva ad est alla corte centrale, sulla quale presumibilmente si affacciavano una serie di ballatoi del piano superiore (è stato infatti identificato il vano cieco che ospitava la scala per il piano superiore ${ }^{15}$.

I lati orientale e meridionale della corte davano accesso rispettivamente alle sale di rappresentanza e al settore privato dell'abitazione: ad est si apriva una sala da ricevimento con ampio ingresso porticato e pavimentazione in intonaco calcareo, fiancheggiata da una cucina a cielo aperto con un forno all'interno, a sua volta adiacente ad un ambiente con funzioni probabilmente di servizio analoghe (fig. 9).

Nella stanza, denominata L.1044, che si apre nell'angolo sud-orientale della corte (fig. 10), sono stati effettuati, nel corso della campagna di scavi del 2003, degli interessanti ritrovamenti, tra i quali l'altare bruciaprofumi oggetto di questo studio. L'ambiente era coperto da uno spesso strato di crollo (US.1034) composto da mattoni crudi e carboni, risultanti dalla violenta conflagrazione che distrusse l'edificio. Tale strato aveva sigillato, schiacciandoli sul pavimento, gli oggetti e gli utensili presenti nella stanza al momento dell'incendio, insieme a quelli conservati nel piano superiore, rinvenuti, infatti, più in alto nello stesso strato di crollo (fig. 11). L’utensile principale rinvenuto in L.1044 era un telaio

Nigro 2004a, 344-346, tavv. XCI-XCII:MD.02.216/31.

Nigro 2004a, tav. XCIV:MD.02.206/9.

Nigro 2001-2003, 567-570; Nigro 2004a, 201-204, tavv. LV-LVIII:MD.02.69.

9 L'arula in terracotta ha forma rettangolare (altezza $0,154 \mathrm{~m}$, base $0,288 \times 0,158 \mathrm{~m}$, spessore $0,28 \mathrm{~m}$ ) con doppia cornice in alto e in basso ed è cava all'interno (Nigro 2004a, tav. LI:MD.02.73).

10 Nigro 2004a, tavv. LII:MD.02.72; XLVII:MD.02.42; XLVII:MD.02.62.

11 Nigro 2004a, tavv. LXXIX, XCVI:MD.02.227/7.

12 Nigro 2004a, 200, nota 111 (MD.02.227/2; MD.02.281/1).

13 Nigro 2004a, 200-202, B.234, tavv. LI, LV-LVIII.

14 Nigro 2004a, 205, fig. 3:59, tav. LIII.

15 Nigro 2004a, 211. 
ligneo verticale da tessitura (fig. 12), di cui rimaneva chiara impronta sul pavimento nella parte occidentale, e resti carbonizzati in quella orientale; vicino, un'anfora punica conteneva i pesi del telaio, 37 in totale, ritrovati sia dentro l'anfora che davanti al contenitore schiacciato (fig. 13). Nell'angolo sud orientale dello stesso ambiente erano invece numerosi vasi a vernice nera di produzione attica e siceliota, soprattutto skyphoi e coppette, nonché alcuni frammenti di ceramica acroma di produzione siceliota, olpai e brocchette (fig. 14).

L'altare bruciaprofumi MD.03.330 (cat. n. 1) era coricato su un lato al centro della stanza. Si tratta di un arredo cultuale realizzato da un blocchetto di calcarenite alto circa $0,19 \mathrm{~m}$, con base quadrata $(0,14 \times 0,18 \mathrm{~m})$ e fusto troncoconico, che presenta un listello centrale sormontato da una cornice a gola egizia e un incavo circolare del diametro di 0,084 $\mathrm{m}$ ricavato nella superficie superiore (fig. 15). È interessante segnalare il rinvenimento, nelle immediate vicinanze dell'altare, delle braccia di una statua in terracotta di grandezza circa 1:3 del vero, con tracce di rivestimento in foglia d'oro ${ }^{16}$, che potrebbe lasciar supporre la presenza di una statuetta cultuale associata al bruciaprofumi, in maniera analoga a quanto raffigurato su alcune stele ${ }^{17}$.

\section{CONFRONTI TIPOLOGICI}

L'altare bruciaprofumi rinvenuto a Mozia si inscrive perfettamente in una tradizione religiosa e cultuale di ambito quasi esclusivamente funerario che vede oggetti di questo tipo ampiamente diffusi nel mondo fenicio orientale e in quello punico come installazioni indipendenti o come elementi compositivi o decorativi nelle stele, e in elementi architettonici pertinenti alla sfera funeraria ${ }^{18}$ : sia nei tofet che nelle necropoli fenicie e puniche sono ampiamente attestati basi-sostegno di idoli/simboli religiosi e sostegni di stele a edicola o di cippi trono ${ }^{19}$, con una forma simile ma una diversa destinazione funzionale ${ }^{20}$, simbolica questi, cultuale e rituale gli altari bruciaprofumi. Ugualmente, per gli altarini rinvenuti nelle necropoli sarde inseriti nel tetto a doppio spiovente delle tombe ${ }^{21}$ si può soltanto ipotizzare una effettiva utilizzazione nell'ambito dei rituali funerari di sepoltura e in onore dei defunti ${ }^{22}$, e pertanto si deve supporre anche per loro una valenza simbolica.

16 Nigro 2004a, 140, tav. XV:MD.03.338; Nigro 2004b, 87, fig. 11.

17 Bisi 1967, n. 1, tav. LVIII; Moscati - Uberti 1981, n. 1010, tav. CLXXXX.

18 Gli altarini bruciaprofumi sono stati oggetto di uno studio tipologico da parte di Giovanni Tore (1989, 109110; 1992, 181).

19 Aimé-Giron 1934, tavv. I-II.

20 Tore 1973, 92, nota 75.

21 Tore 1973, tav. XXVI; 1992, 179-180, nota 9. Nell'area levantina queste installazioni si trovano spesso sui tetti delle abitazioni, dove erano verosimilmente utilizzate per l'offerta di sostanze aromatiche o incenso. Un altare bruciaprofumi rinvenuto ad Ascalona è realizzato in pietra calcarea, ha base quadrata, fusto liscio, cornice aggettante, vasca scavata nella superficie superiore (VII secolo a.C.) (Stager 1996, 66, fig. 8).

22 Nelle stele puniche la stessa tipologia di altari bruciaprofumi è rappresentata a rilievo nel campo figurativo centrale o nella nicchia, dove queste installazioni sono rappresentate nel loro uso come altari indipendenti nel culto, a fianco del personaggio raffigurato; nei cippi e nei cippi-trono i bruciaprofumi sono realizzati a tuttotondo o con un solo lato, quello posteriore, legato ai braccioli che fiancheggiano il simbolo religioso (generalmente l'idolo a bottiglia): in entrambi i casi hanno base quadrata, fusto parallelepipedo leggermente 


\subsection{Mozia e Sicilia}

I bruciaprofumi a gola egizia, rinvenuti in contesti sacri o relativi ad un ambito funerario, sono molto diffusi in tutto il mondo punico tra il VI e il II secolo a.C. Identificati nel loro ruolo funzionale per la presenza di una apposita cavità nella parte sommitale, sono altari di medie e piccole dimensioni, generalmente a basamento troncopiramidale e con modanature nella parte superiore, con apposito spazio alla sommità destinato a rituali ${ }^{23}$, collocati generalmente nelle necropoli, all'interno della tomba o all'esterno, vicino all'ingresso ${ }^{24}$, o nei tofet. Questi arredi potevano essere "a tutto tondo" o addossati alla parete (in questo caso erano lavorati soltanto su tre lati, e il quarto, quello appoggiato al muro, rimaneva appena sbozzato) ${ }^{25}$.

All’interno di questa classe di arredi cultuali così frequentemente attestata nei contesti sopra descritti, quello rinvenuto nella "Casa del sacello domestico" costituisce l'unico esempio finora noto di installazione di questo tipo adibita al culto privato e rinvenuta all’interno di una abitazione nel V secolo a.C.: il tipo di arula domestica più frequentemente attestato nell’isola, rinvenuto, infatti, anche all'interno della "Casa del sacello domestico" stessa tra gli oggetti di culto del sacello domestico L. $300^{26}$, è quello di tradizione siceliota a base rettangolare, fusto parallelepipedo con doppia cornice, in alto e in basso, incavo sulla superficie superiore e raffigurazioni a rilievo sulla faccia anteriore, queste due ultime caratteristiche non sempre presenti ${ }^{27}$.

A Mozia altari bruciaprofumi a gola egizia sono presenti nel tofet nel VI secolo a.C. ${ }^{28}$ sia come installazioni cultuali indipendenti ${ }^{29}$, sia come basi-sostegno di un oggetto di culto (fig. 16): in quest'ultimo caso la superficie superiore è forata da un incasso rettangolare (cat. n. 2) (30 $^{3}$ verosimilmente concepito per l'incastro di un betilo o di un idolo a bottiglia, come suggeriscono reperti di tipologia affine rinvenuti nel tofet di Cartagine ${ }^{31}$. Altarini a

rastremato, toro e corpo superiore conformato a gola egizia con aggiunta, in qualche caso, di una ulteriore vasca rialzata collocata all'interno di tale incavo (Bisi 1967, figg. 9-10, tav. XVIII:1).

23 G. Tore individua due tipologie di altari bruciaprofumi, delle quali la prima (D.1) comprende altari costituiti da un solo elemento, la seconda (D.1/a) arredi con modanatura semplice o multipla (Tore 1999, 417-418).

24 Tore 1999,418

25 Si vuole ricordare, in generale, che le varianti morfologiche degli altari bruciaprofumi “a tuttotondo” o rappresentati a rilievo sulle stele sono molteplici e non sempre attribuibili ad un preciso ambito cronologico, ad una diversa funzione all'interno del culto o a contesti culturali regionali diversi, e che le tre classi sopra enunciate sono categorie dai confini sfumati e assolutamente permeabili; tuttavia si tenterà, prendendo in esame alcuni degli esempi più significativi, di operare una distinzione funzionale di massima che tenga conto di questi fattori e che, proprio per le numerose varianti, consideri che molto spesso la diversa realizzazione dei manufatti non dipende da una intenzionale volontà di differenziare l'oggetto ma dall'abilità e dal gusto dell'artigiano (Tore 1989, 109-110; Tore 1992, 180).

26 Nigro 2004a, 200-201.

27 Famà 1989, 81-82; Belvedere 1982, 67-68.

28 Ciasca 1970, 79-80.

29 Moscati - Uberti 1981, 32-33, nn. 1013, 1014, tav. CLXXXVI.

30 Ciasca 1970, 79-80; Moscati - Uberti 1981, 32-33, n. 1012, tav. CLXXXVI.

31 Bartoloni 1976, n. 30, tav. VIII; anche a Nora (Sardegna) è attestata una base modanata con incasso superiore: Moscati - Uberti 1970, n. 83, tav. XLII. Nel caso specifico della base rinvenuta nel tofet di Mozia, l'incavo sulla superficie superiore potrebbe essere funzionale, per forma e dimensioni, all'incasso di una vaschetta 
gola egizia sono attestati anche nella necropoli punica di Palermo: dagli scavi eseguiti negli anni 1953-54 da Jole Bovio Marconi, e pubblicati da Ida Tamburello nel 196732, provengono tre installazioni cultuali a base troncoconica, cornice aggettante e gola egizia, due delle quali con la variante della doppia modanatura superiore: il primo altare bruciaprofumi (fig. 17) è datato al VI-V secolo a.C., in base ai materiali del contesto di appartenenza, la tomba T.63 (cat. n. 3) 3. $^{33}$, mentre gli altri due costituirebbero esempi più tardi della stessa tipologia: uno di questi (fig. 18, cat. n. 4) è stato rinvenuto all'esterno di una tomba priva di corredo ${ }^{34}$, ed è dunque di datazione incerta, ma è ascrivibile, in base al confronto con un altro rinvenuto nella stessa necropoli di cui parla Bovio Marconi ${ }^{35}$, al III secolo a.C.; l'altro (fig. 19, cat. n. 5) è quasi certamente più tardo, perché pertinente, secondo Tamburello, al periodo di reimpiego della tomba in cui è stato rinvenuto ${ }^{36}$, inquadrabile nella seconda metà del III secolo a.C. Gli scavi condotti nelle necropoli palermitane negli anni '70 e '80 hanno portato al rinvenimento di altri otto altari bruciaprofumi realizzati, come quelli precedentemente descritti, in calcarenite locale e appartenenti alla stessa tipologia ${ }^{37}$.

La celebrazione di culti praticati in ambito domestico, espressione di una religiosità vissuta nel chiuso dell'intimità familiare, è attestata a Solunto dal rinvenimento di questa categoria di reperti all'interno delle abitazioni di età ellenistica e romana: i piccoli altarini bruciaprofumi in calcare stuccato, di dimensioni minuscole e di forme decorative assai semplificate, provengono dalle abitazioni di età ellenistica, dove sono attestati sia il più comune tipo circolare $^{38}$, sia quello quadrato o rettangolare con sponde verticali sulla superficie superiore ${ }^{39}$, che riprende il modello di altare bruciaprofumi a gola egizia (fig. 20, cat. n. 6). Gli scavi alla necropoli di Solunto testimoniano, tuttavia, un utilizzo e una diffusione di queste installazioni, pur se in un ambito differente, quello funerario, anche più antichi (fig. 21) ${ }^{40}$.

\subsection{Africa del Nord}

Numerose sono le attestazioni di questa tipologia in Africa del Nord: dal Santuario di Kerkouane proviene un altare definito "di tipo egizio"41 (fig. 22, cat. n. 7) realizzato in

metallica; in questo caso si tratterebbe dunque di un altare bruciaprofumi simile a quelli rappresentati davanti ai braccioli nei cippi-trono.

32 Tamburello 1967, 362, nota 2, fig. 21.

33 Tomba a camera T.63 (Tamburello 1967, 362, nota 2, 372, fig. 21:a).

34 Tomba a camera T.185 (vuota) (Tamburello 1967, 362, nota 2, 372, fig. 21:c).

35 Forse si tratta dell'altare n. cat. 132, privo di contrassegni ma presumibilmente riferibile allo stesso contesto dei precedenti (Tore 1999, 417).

36 Tomba a camera T.269 (Tamburello 1967, 362, nota 2, 372, fig. 21:b).

37 Tore 1999, 422-423, fig. 427.

38 Hvidberg-Hansen 1984, 25-48.

39 Un altare di questo tipo è conservato al Museo Archeologico Nazionale Baglio Anselmi di Marsala (TP). Il reperto, di provenienza incerta, è attribuito al II secolo a.C. (Spagnoli 2003, 169, nota 8, fig. 5).

40 All'interno dell'Antiquarium del sito è infatti esposto un altare bruciaprofumi con fusto parallelepipedo, privo di cornice, doppia gola egizia e vasca scolpita nella superficie superiore, proveniente dalla necropoli e datato al V-IV secolo a.C.

41 Fantar 1986, 184; tav. XCVI. 
pietra calcarea, simile per dimensioni a quello della "Casa del sacello domestico" di Mozia $^{42}$, mentre un altro bruciaprofumi, rinvenuto nella necropoli di Byrsa (fig. 23, cat. n. 8), presenta una doppia gola egizia e vasca rialzata, e può essere datato, in base ai materiali del corredo funerario di cui era parte, all'inizio del VI secolo a.C. ${ }^{43}$.

La tipologia dell'altare bruciaprofumi con toro e gola egizia è ancor più largamente attestata nel tofet di Cartagine: qui gli altari rinvenuti hanno un'altezza variabile compresa tra $0,5 \mathrm{~m}$ e $1 \mathrm{~m}$, la base generalmente quadrata, il fusto alto e stretto, il toro e la parte superiore diversamente articolata con gola egizia doppia o tripla, con o senza pyramidion o con sponde laterali rialzate (figg. 24-26, cat. nn. 9-11) ${ }^{44}$.

\subsection{Sardegna}

Il panorama sardo offre molte attestazioni della stessa tipologia nei tofet e nelle necropoli ${ }^{45}$, dove, come accennato in precedenza, tali installazioni erano presenti anche come parte (inserita ad incastro) del tetto a doppio spiovente che ricopriva le tombe a camera $^{46}$. A Nora, a Tharros e nelle necropoli del cagliaritano si hanno alcuni esempi di altari bruciaprofumi a toro e gola egizia di dimensioni ridotte rispetto a quelli sopra mostrati dal tofet di Cartagine e analoghe a quelle del bruciaprofumi di Mozia. L'esemplare in pietra tenera proveniente da Nora (fig. 27, cat. n. 12), rinvenuto nel pozzo del Tempio della Dea, detto anche Tempio di Tanit, nel corso degli scavi G. Pesce, presenta tracce di fuoco all'interno del "dadetto" o vasca superiore ${ }^{47}$. Anche nel tofet di Tharros è stata rinvenuta una installazione cultuale morfologicamente affine (fig. 28, cat. n. 13) ${ }^{48}$ interpretata da Tore come altare votivo ${ }^{49}$, proprio come l'altare della "Casa del sacello domestico" e quelli rinvenuti nelle necropoli di Palermo e di Cartagine. Dalla necropoli occidentale di Karalis (fig. 29, cat. n. 14$)^{50}$ e da quella di Sant'Avendrace (fig. 30, cat. n. $15)^{51}$ provengono altri due bruciaprofumi dello stesso tipo.

\subsection{Penisola Iberica}

In Spagna il tipo è rappresentato da tre esemplari, due rinvenuti fuori contesto in alcune tombe a camera della necropoli di Villaricos (figg. 31-32, cat. nn. 16-17) (22 $^{5}$ l'altro in una tomba presso le miniere di Tarsis a Huelva (fig. 33, cat. n. 18) ${ }^{53}$. Questi sono presumibilmente ascrivibili al VI secolo a.C. in base al confronto con gli esemplari nord africani: il primo e il terzo altare, a base quadrata e fusto alto e stretto, sono infatti simili a

42 Per la presenza di installazioni simili in Egitto a partire dal IV-III secolo a.C.: Soukiassian 1983, figg. 16-22.

43 Lancel 1986, 13.

44 Bartoloni 1976, 85, nn. 43, 44, 46, fig. 2, tavv. XII-XIII.

45 Per gli altari bruciaprofumi provenienti dalle necropoli e dai tofet sardi: Tore 1973, tav. XXV; 1989, tav. IV.

46 Tore 1989, tav. I: 20.

47 Tore 1973, nota 81, tav. XIX:2.

48 Moscati - Uberti 1985, 127, n. 167, tav. LXXI

49 Tore 1992,181 , nota 11.

50 Tore 1973, 104-105, tav. XXIV:1.

51 Tore 1973, 105-106, tav. XXIV:2.

52 Astruc 1951, 69, nota 476, tav. L:1, 4

53 García y Bellido 1942, 294, fig. 57. 
quelli rinvenuti nel tofet di Cartagine e nelle necropoli puniche della Sardegna, mentre il secondo è più piccolo e di forma leggermente variata, privo di toro e con gola egizia sormontata da elemento a parallelepipedo in pietra.

\subsection{Il Levante e Cipro}

Il tipo dell'altare bruciaprofumi a gola egizia è dunque ampiamente diffuso nel mondo punico sin dal VII secolo a.C., come mostrano i ritrovamenti più antichi del tofet di Cartagine, ma trova antecedenti nell'area siro-palestinese dove, fin dal X secolo a.C., è diffuso un tipo di altare a corna attestato nei centri fenici di Sidone e Tiro nella forma di base-sostegno di stele ${ }^{54}$, e nelle necropoli e nei santuari ciprioti e levantini.

Nella regione siro-palestinese altari bruciaprofumi a base rettangolare o quadrata, con alto fusto liscio rastremato verso l'alto, cornice aggettante e parte superiore con vaschetta scavata nella superficie o munita di corna angolari, sono ampiamente attestati nei contesti sacri: si ricordano gli altari con corna angolari rinvenuti a Megiddo ${ }^{55}$, a Tell Kedesh ${ }^{56}$, i due altari bruciaprofumi collocati all'ingresso dell'“Alto Luogo" di Arad ${ }^{57}$, i numerosi altari rinvenuti fuori contesto nel santuario di Eshmun a Sidone (fig. 35, cat. nn. 19-21) ${ }^{58}$, i due altari rinvenuti nel Santuario dello "Ingot God" ad Enkomi (Cipro), del XII secolo a.C. (cat. nn. 22-23) ${ }^{59}$, gli altari rinvenuti dagli archeologi della missione francese a Kition-Bamboula (cat. nn. 24-25) ${ }^{60}$.

Rispetto ai più comuni altari bruciaprofumi rinvenuti in contesti sacri nel mondo punico e in quello fenicio orientale, l'arredo di Mozia presenta dimensioni diverse ed è più piccolo di circa un terzo: si può quindi tentare, in linea ipotetica e in assenza di puntuali confronti nel mondo punico con altri provenienti da contesti domestici, una distinzione tra un tipo "ufficiale" e collettivo di altare bruciaprofumi con fusto alto e stretto, cornice a circa tre quarti dell'altezza e gola egizia poco elevata rispetto a questo ${ }^{61} \mathrm{e}$ un tipo destinato al culto domestico "privato" o di natura votiva ${ }^{62}$, di dimensioni minori e con un diverso rapporto proporzionale tra gli elementi compositivi ${ }^{63}$. Questa distinzione si riscontra anche nell’area

54 Bisi 1967, tavv. I, II:1.

55 May - Engberg 1935, tav. XII:10, 11; Loud 1948, tav. 254:1-2 (X secolo a.C.). Un altare con corna di forma analoga a quelli in pietra, ma in terracotta, è stato rinvenuto in un pozzo votivo individuato fortuitamente nel 2002 presso Yavneh. I materiali della favissa erano probabilmente pertinenti a un edificio di culto e sono databili tra la fine del Tardo Bronzo e la prima età del Ferro (Vieweger 2012, 476-478, tav. 66:B).

62 Come già affermato da G. Tore (1992, 181, nota 11). A questo gruppo sarebbero ascrivibili: l'altare egittizzante di Kerkouane, quello dalla tomba L.13 di Byrsa, il bruciaprofumi stuccato di Solunto, quelli dalla necropoli di Palermo, l'altare 167 del tofet di Tharros e l'altare dal pozzo del tempio di Tanit a Nora, i due provenienti dalle necropoli di Cagliari (Karalis e Sant'Avendrace), e l'altare più piccolo dei due rinvenuti nella necropoli di Villaricos.

63 Non sempre questo si verifica: vedi il piccolo bruciaprofumi di Byrsa o quello rinvenuto nella Corte II del santuario di Kerkouane: il fattore che rimane costante è la diminuzione delle dimensioni. 
siro-palestinese e cipriota, dove molti degli altari con le corna rinvenuti ad Ekron-Khirbet el-Muqanna', datati al VII-VI secolo a.C., rinvenuti nella zona industriale del sito, negli ambienti adibiti alla molitura delle olive ${ }^{64}$, sono, rispetto ai più antichi esemplari di Megiddo e della zona settentrionale della costa siro-palestinese, e rispetto a quelli originariamente collocati in luoghi di culto, più bassi e larghi, con cornice arrotondata e parte superiore spesso priva di corna; di dimensioni ridotte sono anche tre altari, portati alla luce durante gli scavi al santuario di Kharayeb effettuati alla fine degli anni ' $60^{65}$ e datati con probabilità al VI secolo a.C. La stessa osservazione trova riscontro anche negli altari bruciaprofumi rinvenuti fuori contesto nella rampa d'assedio del Santuario di Afrodite a Kouklia-Palaepaphos (fig. 34$)^{66}$, per i quali si può ipotizzare una funzione simile.

\section{CONCLUSIONI}

A seguito di quanto detto, è possibile dunque affermare che la tipologia di arredo cultuale rappresentata dall'altare bruciaprofumi della "Casa del sacello domestico” a Mozia sia un prodotto derivato da un modello levantino ampiamente diffuso nelle culture del Mediterraneo orientale, che si installa nell'isola fin dall'epoca arcaica e che si perpetua pressoché invariato nelle tradizioni religiose e figurative locali e più in generale del mondo punico.

Circa la specifica funzione dell'altare bruciaprofumi della "Casa del sacello domestico" si possono avanzare alcune ipotesi interpretative che, nonostante la mancanza di dati più precisi sull'esatto contesto di rinvenimento di molti degli altari punici sopra citati provenienti sia da necropoli che da aree sacre, sono suffragate tuttavia dai dati provenienti dall'analisi del contesto stratigrafico di rinvenimento dell'installazione all'interno dell'abitazione e della ricostruzione della vita della residenza moziese.

La prima considerazione riguarda appunto il luogo di rinvenimento dell'altare: probabilmente l'ambiente L.1044 non era la collocazione originaria dell'arredo, dal punto di vista funzionale; nel contesto sigillato dal crollo non è infatti stato individuato alcun tipo di supporto ${ }^{67}$, sia ligneo che di altro materiale, su cui questo sarebbe dovuto essere collocato $^{68}$, insieme alla statua di culto; si può quindi ipotizzare che l'altare bruciaprofumi sia stato spostato in L.1044, una delle stanze più interne e quindi protette dell'edificio, forse poco prima della violenta distruzione dell'edificio del 397/6 a.C., magari prelevandoli dal

64 Gitin 1989, 57-67; 1992, 43-49.

65 Si tratta di altari in pietra tenera locale, frammentari e molto danneggiati, rispettivamente delle dimensioni di: $0,49 \times 0,31 \mathrm{~m} ; 0,30 \times 0,19 \mathrm{~m} ; 0,29 \times 0,305 \mathrm{~m}$. L'altare meglio conservato presenta base quadrata, fusto leggermente rastremato, cornice a sezione rettangolare e parte superiore a blocco con incavo rettangolare sulla superficie superiore. La datazione è fissata al VI secolo a.C. Nel rapporto di scavo non si danno indicazioni più precise circa l'originaria collocazione e la funzione di queste installazioni; è probabile, comunque, che si tratti non di installazioni cultuali ma, più verosimilmente, di oggetti votivi (Kaoukabani 1973, 41-42, 54, tav. XVIII:3).

66 Questi reperti sono datati al VI-V secolo a.C.

67 Al contrario, i resti lignei carbonizzati del telaio (con relativi chiodi in bronzo) sono stati rinvenuti ben visibili in impronta sul pavimento L.1044 coperto dal crollo.

68 Gli oggetti cultuali in L.300 erano collocati su di un blocco di pietra (Nigro 2004a, 200, figg. 3.49-3.50; 2004b, 86, figg. 6-7, 15). 
vicino sacello domestico.

La seconda osservazione riguarda la specifica funzione dell'oggetto nell'ambito dei culti domestici comunemente praticati. Come è emerso dagli esempi di altari bruciaprofumi noti, nel mondo punico l'uso di questo tipo di installazioni è connesso ad un ambito funerario; anche nel caso si tratti di oggetti votivi, questi sono comunque legati alla sfera funeraria, sia nel caso facciano parte del corredo funebre, sia che si trovino in santuari. Per questo oggetto si potrebbe ipotizzare, tuttavia, non tanto un uso come supporto per la combustione di sostanze aromatiche, erbe o incensi, ma piuttosto un utilizzo nel culto libatorio praticato in casa e dedicato ai defunti69: l'altare bruciaprofumi di Mozia non presenta, infatti, segni di bruciato sulla superficie superiore e proprio nei pressi dell'altare sono stati rinvenuti (pur se in collocazione probabilmente non originaria), raggruppati nell'angolo sud occidentale della stanza, vasi potori a vernice nera connessi verosimilmente alla pratica del culto.

\section{CATAlOgO}

Cat. n. 1 (fig. 15): Mozia, Zona D, “Casa del sacello domestico”. Materiale: calcarenite. Dimensioni: altezza 0,19 circa; base 0,14 x 0,18 m; diametro incavo superiore 0,084. Descrizione: arredo cultuale a base quadrata e fusto troncoconico terminante con un listello a sezione rettangolare centrale sormontato da una cornice a gola egizia. Nella superficie superiore è ricavato un incavo circolare. Frammentario. Datazione: V secolo a.C. Bibliografia: Nigro 2007a, 138, tav. XIV:MD.03.330.

Cat. n. 2 (fig. 16): Mozia, tofet, strato IV, muro T2, n. inv. S281. Materiale: calcarenite. Dimensioni: altezza 0,22 m; larghezza 0,32 m; spessore: 0,20 m. Descrizione: sostegno a base rettangolare sagomata a plinto sormontato da gola egizia su listello a sezione rettangolare. Sulla superficie superiore, foro rettangolare d'incastro $(0,07 \times 0,06$ x 0,085 m). Integro. Datazione: metà VI-V secolo a.C. Bibliografia: Ciasca 1970, 79-80; Moscati - Uberti 1981, 32-33, n. 1012, tav. CLXXXVI.

Cat. n. 3 (fig. 17): Palermo, necropoli, tomba T.63 (saggio VI, 2-XI-1953), S1, n. inv. 33797. Materiale: calcarenite. Dimensioni: altezza 0,355 m; base inferiore 0,22 x 0,17 m; base superiore $0,145 \times 0,14 \mathrm{~m}$. Descrizione: altare bruciaprofumi a base rettangolare, fusto rastremato, doppia gola egizia, incavo nella superficie superiore. La parte inferiore era probabilmente interrata. La superficie superiore presenta delle macchie scure, forse tracce di bruciato. All'altare bruciaprofumi era associato un corredo ceramico arcaico. Integro. Datazione: VI-V secolo a.C. Bibliografia: Tamburello 1967, 362, nota 2, 372, fig. 21:a.

Cat. n. 4 (fig. 18): Palermo, necropoli, tomba T.185 (saggio XVIII, 3-XII-1953), S10, n. inv. 2875. Materiale: calcarenite. Dimensioni: altezza 0,445 m. Descrizione: altare bruciaprofumi con parte inferiore cubica, base sottostante aggettante di 0,003 m circa, originariamente interrata, doppia gola egizia e incavo profondo sulla superficie superiore. Parte posteriore non lavorata. Abraso. Datazione: III secolo a.C. Bibliografia: Tamburello 1967, 362, nota 2, 372, fig. 21:c.

Cat. n. 5 (fig. 19): Palermo, necropoli, tomba T.69 (saggio XIX, 19-V-1954), S2, n. inv. 33798. Materiale: calcarenite. Dimensioni: altezza 0,245 m; base inferiore 0,08 x 0,10 m; base superiore 0,11 x 0,13 m. Descrizione: altare bruciaprofumi a base rettangolare, fusto rastremato verso il basso, doppia gola egizia, incavo nella superficie superiore. Abraso e rotto nella parte inferiore. Datazione: III secolo a.C. Bibliografia: Tamburello 1967, 362, nota 2, 372, fig. 21:b.

69 Tore 1999, 417 
Cat. n. 6 (fig. 20): Solunto, abitato. Materiale: calcare bianco. Dimensioni: altezza 0,20 m circa. Descrizione: altarino bruciaprofumi a fusto parallelepipedo in calcare ricoperto di stucco bianco, cornice e parte superiore con sponde rialzate, al centro delle quali vi sono evidenti tracce di bruciato. Integro. Datazione: II-I secolo a.C.

Cat. n. 7 (fig. 22): Kerkouane, Santuario, Corte II. Materiale: calcare tenero. Dimensioni: altezza 0,221 m; altezza fusto 0,107 m; base quadrata 0,14 m (inf.); 0,128 m (sup.). Descrizione: altarino bruciaprofumi composto da un supporto troncopiramidale a base quadrata, listello a sezione rettangolare e gola egizia soprastante. Frammentario. Datazione: IV-III secolo a.C. Bibliografia: Fantar 1986, 184; tav. XCVI.

Cat. n. 8 (fig. 23): Cartagine, necropoli di Byrsa, tomba L.13. Materiale: calcare. Dimensioni: altezza 0,15 m. Descrizione: altarino bruciaprofumi (Petit pilier “djed”) a base rettangolare con fusto rastremato verso l'alto, toro e doppia gola egizia. Sulla superficie superiore è una vasca con incavo rettangolare all'interno della quale sono visibili tracce di bruciato. Angolo del toro e vasca superiore danneggiati. Abraso. Datazione: inizi VI secolo a.C. Bibliografia: Lancel 1986, 13.

Cat. n. 9 (fig. 24), Cartagine, tofet. Materiale: arenaria giallastra. Dimensioni: altezza 0,58 m; base $0,22 \times 0,18 \mathrm{~m}$; altezza rilievo $0,03 \mathrm{~m}$. Descrizione: altare bruciaprofumi a base rettangolare, fusto rastremato, cornice aggettante e gola egizia. Abraso. Angoli inferiori mancanti. Datazione: VIIVI secolo a.C. Bibliografia: Bartoloni 1976, 85, n. 43, tav. XII.

Cat. n. 10 (fig. 25), Cartagine, tofet. Materiale: arenaria giallastra. Dimensioni: altezza 0,79 m; base 0,225 x 0,19 m; altezza rilievo 0,025 m. Descrizione: altare bruciaprofumi a base rettangolare, fusto leggermente rastremato, cornice aggettante e gola egizia con lieve incavo sulla superficie superiore. Abraso. Datazione: VII-VI secolo a.C. Bibliografia: Bartoloni 1976, 85, n. 44, tav. XII.

Cat. n. 11 (fig. 26), Cartagine, tofet. Materiale: arenaria giallo-rosata. Dimensione: altezza 0,59 $\mathrm{m}$; base 0,175 x 0,175 m; altezza rilievo 0,055 m. Descrizione: altare bruciaprofumi a base quadrata, fusto dritto, cornice e gola egizia molto aggettante con pyramidion alla sommità. Abraso, punta del pyramidion mancante. Datazione: VII-VI secolo a.C. Bibliografia: Bartoloni 1976, 85, n. 46, fig. 2, tav. XIII.

Cat. n. 12 (fig. 27): Nora, Tempio di Tanit. Materiale: calcare. Dimensioni: altezza 0,14 m; base 0,06 x 0,055 m; aggetto della base $0,01 \mathrm{~m}$; altezza della base $0,02 \mathrm{~m}$ circa; aggetto della cornice inferiore $0,01 \mathrm{~m}$; larghezza della cornicetta superiore $0,01 \mathrm{~m}$; dimensioni del dadetto $0,075 \times 0,07 \mathrm{~m}$; altezza del dadetto 0,02 m; massima profondità del dadetto 0,015 m. Descrizione (da Tore 1973, 90): altare bruciaprofumi con base a sezione quadrangolare, con doppia risega residua solo in parte su un lato. La parte superiore della base è leggermente spiovente verso il basso e aggetta rispetto alla parte centrale [...]. Parte centrale parallelepipeda con tracce di graffiti su ogni lato. Pare potersi individuare un motivo a festoni molto stilizzato (triangolari, a più linee convergenti verso l'alto), chiaramente, almeno su due lati, mentre negli altri residuano solo tracce indistinte [...]. Al di sopra del parallelepipedo aggetta una cornice che descrive una forma piuttosto dolce (tipo "a gola egizia" piuttosto semplificata) che è sormontata da un'altra cornice "a gola egizia". Le due cornici sono sormontate da due modanature piatte. La sommità presenta un dadetto rilevato circondato su ogni lato da una cornicetta lievemente inclinata verso il basso. All'interno il dadetto è cavo [...]. Tracce di fuoco sui bordi del dadetto. Soltanto un lato è conservato integralmente; abraso su tutta la superficie. Datazione: III secolo a.C. Bibliografia: Tore 1973, 90, nota 81, tav. XIX:2.

Cat. n. 13 (fig. 28): Tharros, tofet, altare 167. Materiale: arenaria eolica. Dimensioni: altezza 0,17 $\mathrm{m}$; base $0,11 \times 0,10 \mathrm{~m}$. Descrizione: altare sagomato a plinto sormontato da gola egizia su listello. Integro. Datazione: VI-inizi IV secolo a.C. Bibliografia: Moscati - Uberti 1985, 127, n. 167, tav. LXXI. 
Cat. n. 14 (fig. 29): Cagliari, Predio Ibba, necropoli occidentale di Karalis, T.63 (pozzo di accesso), n. inv. 32922. Materiale: calcare. Dimensioni: altezza 0,23 m; larghezza base 0,15 m, sommità $0,115 \mathrm{~m}$, listello 0,105 m; spessore $0,09 \mathrm{~m}$; aggetto del listello 0,02 m. Descrizione: altare a fusto troncopiramidale, toro a sezione rettangolare molto aggettante, gola egizia e vasca superiore con incavo rettangolare. Fortemente danneggiato alla base e alla sommità. Datazione: IV-III secolo a.C. Bibliografia: Tore 1973, 104-105, tav. XXIV:1.

Cat. n. 15 (fig. 30): Cagliari, Necropoli di Sant'Avendrace, n. inv. 43510. Materiale: calcare. Dimensioni: altezza: 0,155 m; larghezza base 0,08 m, sommità 0,063 m, listello 0,105 m; spessore $0,07 \mathrm{~m}$; aggetto del listello $0,01 \mathrm{~m}$. Descrizione: altare a fusto troncopiramidale, toro a sezione rettangolare molto aggettante, gola egizia e vasca superiore con incavo rettangolare. Fortemente danneggiato alla base e alla sommità; incompleto. Datazione: V-IV secolo a.C. Bibliografia: Tore 1973, 105-106, tav. XXIV:2.

Cat. n. 16 (fig. 31): Villaricos, necropoli. Materiale: calcare. Descrizione: altare bruciaprofumi a base rettangolare, fusto rastremato, toro e gola egizia. Datazione: incerta, probabilmente VI secolo a.C. Bibliografia: Astruc 1951, 69, nota 476, tav. L:1.

Cat. n. 17 (fig. 32): Villaricos, necropoli. Materiale: calcare. Descrizione: altare bruciaprofumi a base rettangolare, fusto fortemente rastremato, cornice aggettante e vasca rialzata sulla superficie superiore. Datazione: incerta, probabilmente VI secolo a.C. Bibliografia: Astruc 1951, 69, nota 476, tav. L:4.

Cat. n. 18 (fig. 33): Huelva, sepoltura presso le miniere di Tarsis, contesto di rinvenimento incerto. Materiale: calcare. Dimensioni: altezza 1,0 m; base 0,63 m. Descrizione: base quadrata, fusto troncopiramidale, cornice aggettante a sezione rettangolare, gola egizia. Abraso. Datazione: incerta, probabilmente VI secolo a.C. Bibliografia: García y Bellido 1942, 294, fig. 57.

Cat. n. 19 (fig. 35): Sidone, Bostan es-Sheikh. Materiale: calcare. Dimensioni: altezza 0,88 m; base $0,22 \mathrm{~m}$; installazione a base quadrata, con fusto troncoconico, cornice listello a sezione rettangolare, gola egizia soprastante, con rialzo sulla superficie superiore. Completo. Datazione: incerta, probabilmente VI secolo a.C. Bibliografia: Stucky 1993, 75, n. 62, fig. 5.

Cat. n. 20 (fig. 35): Sidone, Bostan es-Sheikh. Materiale: calcare. Dimensioni: altezza 0,70 m; base $0,29 \mathrm{~m}$. Descrizione: installazione a base quadrata, con fusto troncoconico, cornice listello a sezione rettangolare, gola egizia soprastante con incavo circolare sulla superficie superiore. Completo. Datazione: incerta, probabilmente VI secolo a.C. Bibliografia: Stucky 1993, 75, n. 63, fig. 5, tav. 16.

Cat. n. 21 (fig. 35): Sidone, Bostan es-Sheikh. Materiale: calcare. Dimensioni: altezza 0,70 m; base $0,29 \mathrm{~m}$. Descrizione: installazione a base quadrata, fusto parallelepipedo, cornice listello a sezione rettangolare poco aggettante, parte superiore conformata a capitello dorico, superficie superiore con incavo circolare. Completo. Datazione: incerta, probabilmente VI secolo a.C. Bibliografia: Stucky 1993, 75, n. 64, fig. 5, tav. 16.

Cat. n. 22: Enkomi (Cipro), “Autel inferieur”, Santuario dello "Ingot God”, corte. Materiale: calcare. Dimensioni: altezza 0,80 m; base 0,47 x 0,32 m. Descrizione: altare bruciaprofumi a base rettangolare e fusto rastremato verso l'alto. Datazione: XII secolo a.C. Bibliografia: Courtois 1971, 186, 190, figg. 30-35.

Cat. n. 23: Enkomi (Cipro), “Autel superieur”, Santuario dello "Ingot God”, corte. Materiale: calcare. Dimensioni: altezza 0,72 m; base: 0,47 x 0,29 m. Descrizione: altare bruciaprofumi a base rettangolare e fusto rastremato verso l'alto. Datazione: XII secolo a.C. Bibliografia: Courtois 1971, 186, 190, figg. 30-35.

Cat. n. 24: Kition-Bamboula, Altare 258. Materiale: calcare. Dimensioni: altezza 0,85 m; base 0,30 x 0,20 m. Descrizione: altare bruciaprofumi a base rettangolare, fusto liscio rastremato verso l'alto, vaschetta rettangolare scavata nella superficie superiore. Integro. Datazione: IX secolo a.C. Bibliografia: Yon - Raptou 1991, tav. III:b. 
Cat. n. 25: Kition-Bamboula, Altare 38. Materiale: calcare. Dimensioni: altezza 1,0 m; base 0,60 x 0,62 m. Descrizione: altare bruciaprofumi a base rettangolare, toro a sezione circolare. Parte superiore mancante. Datazione: IV secolo a.C. Bibliografia: Yon - Raptou 1991, tav. III:c, f.

\section{BIBLIOGRAFIA}

AHARONI, Y.

$1967 \quad$ Excavations at Tell Arad: Israel Exploration Journal 17 (1967), pp. 233-249.

AIMÉ-GIRON, N.

1934 Un naos phénicien de Sidon: Bulletin de l'Institut Français d'Archéologie Orientale 34 (1934), pp. 31-42.

Astruc, $\mathrm{M}$.

1951 La Necropolis de Villaricos (Informes y Memorias 25), Madrid 1951.

BARTOLONI, P.

$1976 \quad$ Le stele arcaiche del tophet di Cartagine (Collezione di Studi Fenici 8), Roma 1976.

BELVEDERE, O.

1982 Tipologia e analisi delle arule imeresi: N. ALLEGRO - O. BELVEDERE - N. BONACASA - J. DE WAele - C.A. Di Stefano - A. Gulì - V. Tusa - A. Tusa Cutroni (a cura di ), Secondo Quaderno Imerese (Studi e Materiali. Istituto di Archeologia - Università di Palermo 3), Roma 1982, pp. 61-113.

BISI, A.M.

$1967 \quad$ Le stele puniche (Studi Semitici 27), Roma 1967.

CIASCA, A.

1970 Lo scavo del 1979: A. CiasCa - M.G. Guzzo Amadasi - S. Moscati - V. TusA, Mozia VI. Rapporto preliminare della Missione congiunta con la Soprintendenza alle Antichità della Sicilia Occidentale (Studi Semitici 37), Roma 1970, pp. 65-81.

COURTOIs, J.-C.

1971 Le Sanctuaire du Dieu au Lingot d'Enkomi-Alasia: C.F.A. SCHAEFFER ET ALII, Alasia I. Première Série publiée à l'occasion de la XX campagne de Fouilles à Enkomi-Alasia (1969) sous la direction de C.F.A. Schaeffer, Paris 1971, pp. 151-362.

FAMÀ, M.L.

1989 Arule e louteria: A. CiASCA ET ALII, Mozia (Itinerari IV), Roma 1989, pp. 81-82.

FANTAR, M.H.

1986 Kerkouane, cité punique du Cap Bon (Tunisie), Tome III, Sanctuaires et cultes. Sociétéeconomie, Tunis 1986.

GARCíA Y BELLIDO, A.

$1942 \quad$ Fenicios y Cartagineses en Occidente, Madrid 1942.

GITIN, S.

1989 Incense Altars from Ekron, Israel and Judah: context and typology: Eretz Israel 20 (1989), pp. 57-67.

1992 New Incense Altars from Ekron: context, typology and function: Eretz Israel 23 (1992), pp. 43-49.

HVIDBERG-HANSEN, F.O.

1984 Due arule fittili di Solunto: Analecta Romana 13 (1984), pp. 25-48.

KAOUKABANI, B.

1973 Rapport préliminaire sur les fouilles de Kharayeb, 1969-1970: Bulletin du Musée de Beyrouth 26 (1973), pp. 41-60. 
LANCEL, S.

1986 Equipe Punique. Rapport sur les travaux d'une mission de courte durée, fin juin-débout juillet 1985: Centre d'études et de documentation archéologique de la Conservation de Carthage. Bulletin 7 (1986), pp. 12-14.

LOUD, G.

1948 Megiddo II. Seasons of 1935-1939 (Oriental Institute Publications 62), Chicago 1948.

MAY, H.G. - ENGBERG, R.M.

1935 Materials remains of the Megiddo Cult (Oriental Institute Publications 41), Chicago 1935. Moscati, S. - UBERTI, M.L.

1970 Le stele puniche di Nora nel Museo Nazionale di Cagliari (Pubblicazioni del Centro di Studi per la Civiltà Fenicia e Punica 5), Roma 1970.

1981 Scavi a Mozia. Le stele (Pubblicazioni del Centro di Studi per la Civiltà Fenicia e Punica, 23 [Serie Archeologica 25]), Roma 1981.

1985 Scavi al tofet di Tharros. I monumenti lapidei (Collezione Studi Fenici 21), Roma 1985.

NigRO, L.

2001-2003 Un arredo in terracotta conformato a capitello eolico da Mozia: Scienze dell'Antichità. Storia, archeologia, antropologia 11 (2001-2003), pp. 567-577.

2003 Nuovi scavi a Mozia dell’Università di Roma «La Sapienza» (XXII campagna, agostoottobre 2002): Sicilia Archeologica 101 (2003), pp. 85-98.

2004a L. Nigro (a cura di), Mozia - X. Rapporto preliminare della XXII campagna di scavi 2002 condotta congiuntamente con il Servizio Beni Archeologici della Soprintendenza regionale per i Beni Culturali e Ambientali di Trapani (Quaderni di Archeologia FenicioPunica I), Roma 2004.

2004b Le pendici occidentali dell’Acropoli (Zona D): la “Casa del sacello domestico”: L. NigRO - G. Rossoni (a cura di), «La Sapienza» a Mozia. Quarant'anni di ricerca archeologica, 1964-2004. Catalogo della Mostra, Università di Roma «La Sapienza», Facoltà di Scienze Umanistiche, Museo dell'Arte Classica, Roma 27 febbraio - 18 maggio 2004 (Quaderni di Archeologia Fenicio-Punica CM/01), Roma 2004, pp. 84-89.

2007a L. Nigro (a cura di), Mozia - XII. Zona D. La "Casa del sacello domestico", il "Basamento meridionale" e il Sondaggio stratigrafico I. Rapporto preliminare delle campagne di scavi XXIII e XXIV (2003-2004) condotte congiuntamente con il Servizio Beni Archeologici della Soprintendenza Regionale per i Beni Culturali e Ambientali di Trapani (Quaderni di Archeologia Fenicio-Punica III), Roma 2007.

2007b Motyé et les récentes découvertes de l'université de Rome «La Sapienza»: Les Dossier d’Archéologie - Hors série 13 (Novembre 2007), pp. 50-55.

2009 Recenti scoperte dell’Università di Roma «La Sapienza» a Mozia (2002-2006): il Tempio del Kothon, la 'Casa del sacello domestico', il 'Basamento meridionale' e la Fortezza Occidentale: C. Ampolo (ed.), Immagine e immagini della Sicilia e di altre isole del Mediterraneo antico. Atti delle seste giornate internazionali di studi sull'area elima e la Sicilia occidentale nel contesto mediterraneo, Erice, 12-16 ottobre 2006, Pisa 2009, pp. 551-559.

SOUKIASSIAN, G.

1983 Les autels 'à cornes' ou 'à acrotères' en Egypte: Bulletin de l'Institut Français d’Archéologie Orientale 83 (1983), pp. 317-333.

SPAGNOLI, F.

2003 Altari punici nei santuari della Sicilia Occidentale: Sicilia Archeologica 101 (2003), pp. 169-190.

STAGER, L.E.

1996 Ashkelon and the archaeology of destruction: Eretz Israel 25 (1996), pp. 61-74. 
STERN, E. - BEIT-ARIEH, I.

$1979 \quad$ Excavations at Tell Kedesh: Tel Aviv 6 (1979), pp. 1-7.

STUCKY, R.A.

1993 Die Skulpturen aus dem Eshmun-Heiligtum bei Sidon. Griechische, römische, kyprische und phönizische Statuen und Reliefs vom 6.Jahrhundert vor Chr bis zum 3. Jahrhundert nach Chr (Siebzehntes Beiheft zur Halbjharesscherift Antike Kunst), Basel 1993.

TAMBURELLO, I

1967 Palermo. La necropoli: l’esplorazione 1953-54: Notizie degli scavi di Antichità XXI (1967), pp. 354-378.

TORE, G.

1973 Due cippi-trono dal tophet di Tharros: Studi Sardi 22 (1971-1972) [1973], pp. 2-152.

1989 Cippi, altarini e stele funerarie nella Sardegna fenicio-punica (nota preliminare): P. BERNARDINI (a cura di), Riti funerari e olocausto nella Sardegna fenicia e punica. Atti dell'incontro di studio, Sant'Antioco 3-4 ottobre 1986 (Quaderni 6/1989 - Supplemento), Cagliari 1989, pp. 109-117.

1992 Cippi, altarini e stele funerarie nella Sardegna fenicio-punica: alcune osservazioni preliminari ad una classificazione tipologica: AA.VV., Sardinia Antiqua. Studi in onore di Piero Meloni in occasione del suo settantesimo compleanno, Cagliari 1992, pp. 177-194.

1999 Cippi, altarini, stele e arredi: C.A. Di Stefano (a cura di), Palermo Punica. Museo Archeologico regionale Antonino Salinas, 6 dicembre 1995 - 30 settembre 1996, Palermo 1999, pp. 417-427.

VIEWEGER, D.

2012 Die Kultausstattung “philistaïscher” Heiligtümer in Palästina: J. KAMLAH (ed.), Temple Building and Temple Cult. Architecture and Cultic Paraphernalia of Temples in the Levant (2.-1. Mill. B.C.E.). Proceedings of a Conference on the Occasion of the $50^{\text {th }}$ Anniversary of the Institute of Biblical Archaeology at The University of Tübingen (28-30 May 2010) (Abhandlungen des Deutschen Palästina-Vereins, Band 41), Wiesbaden 2012, pp. 459-481.

YON, M. - RAPTOU, E.

1991 Autels de Cypre: R. ETIENNE (éd.), L'espace sacrificiel dans les civilisations méditerranéennes de l'antiquité. Actes du colloque, Lyon 4-7 juin 1988 (Publications de la Bibliothèque Salomon-Reinach 5), Lyon 1991, pp. 167-173. 


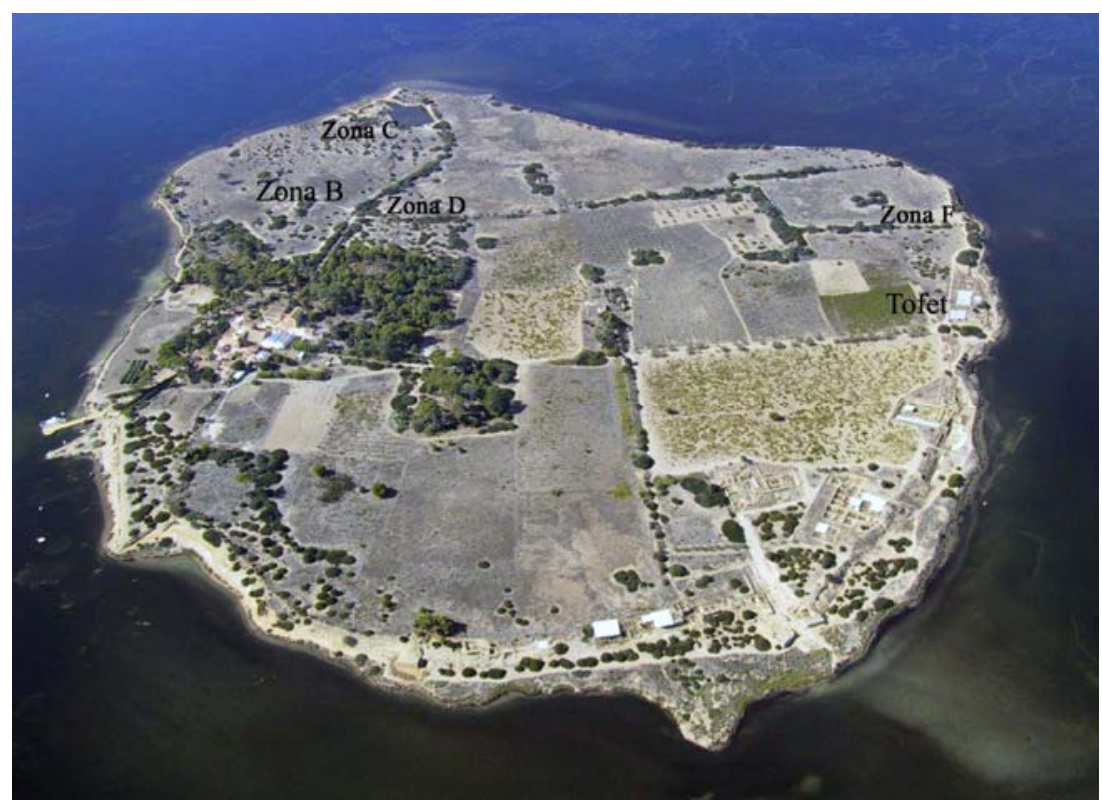

Fig. 1: Mozia (TP), foto aerea con indicazione delle aree interessate dalle attività di scavo della Missione Archeologica dell’Università di Roma «La Sapienza».

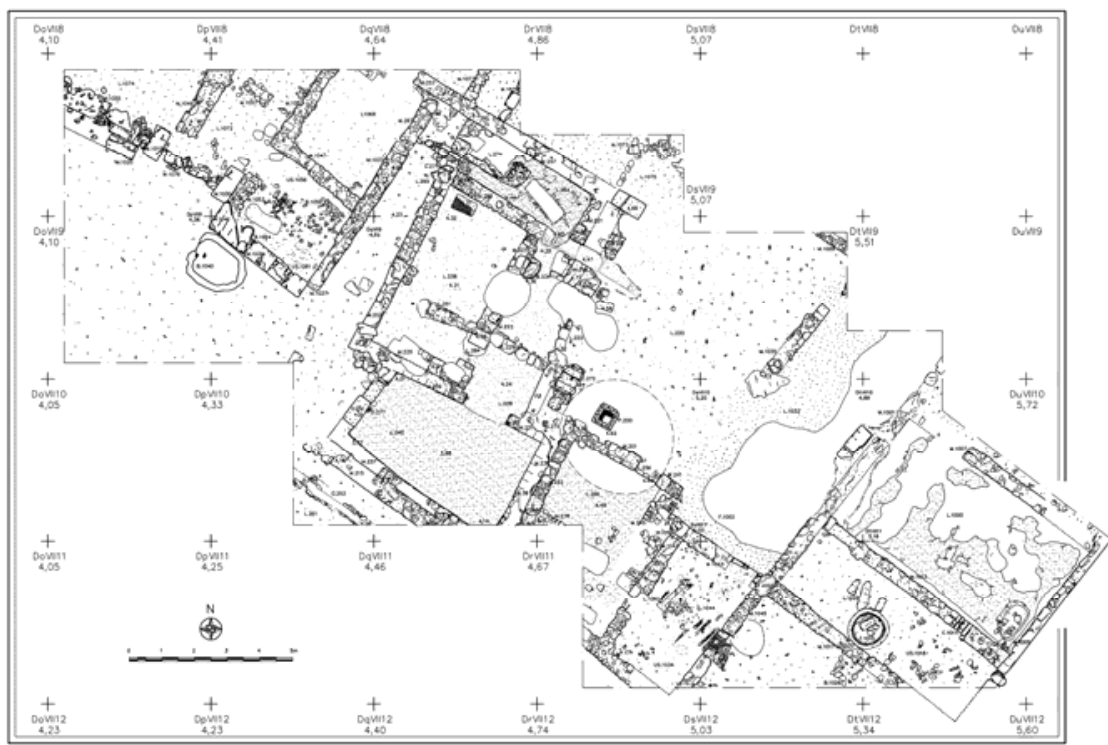

Fig. 2: Pianta dettagliata della "Casa del sacello domestico", Zona D (da Nigro 2007a, 31, fig. 2:24). 


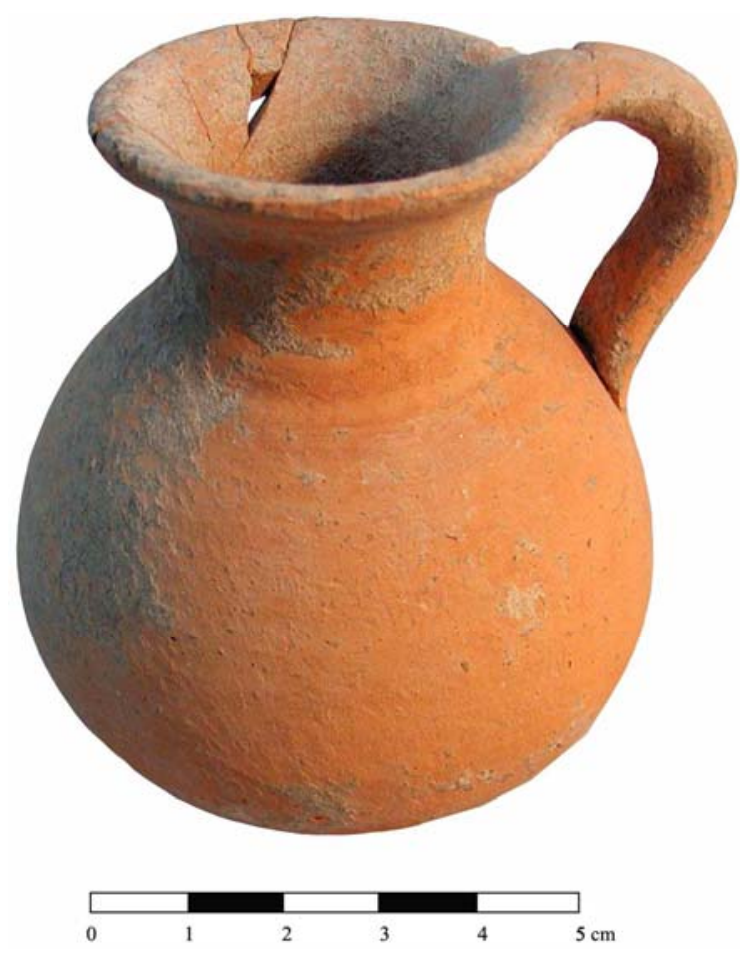

Fig. 3: Olpe in ceramica comune MD.02.277/1 (da Nigro 2004a, tav. LXXXVII).

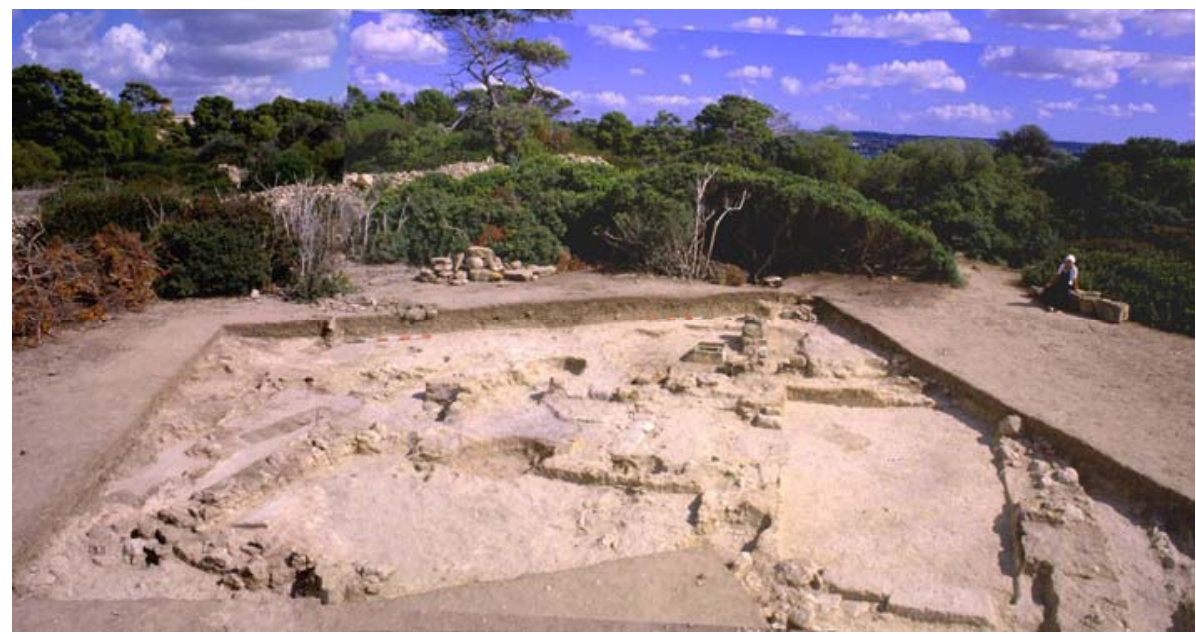

Fig. 4: "Casa del sacello domestico", da ovest; in primo piano, soglia e sala di ingresso con pavimento in marna calcarea pressata e argilla. 


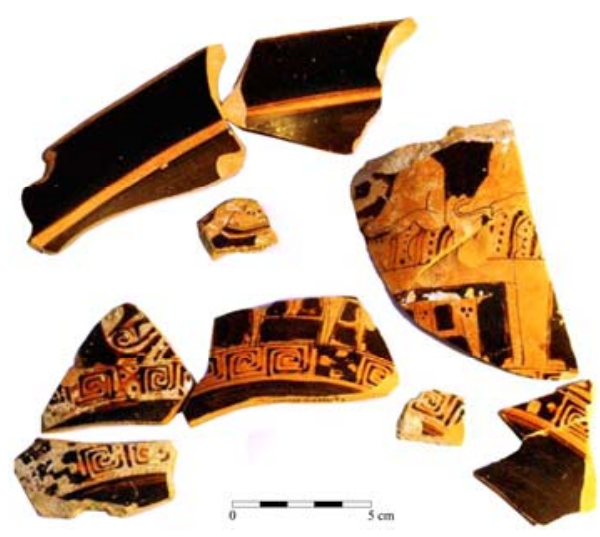

Fig. 5: Cratere a figure rosse, Pittore di Meleagro (da Nigro 2004a, 344, tav. XCII).

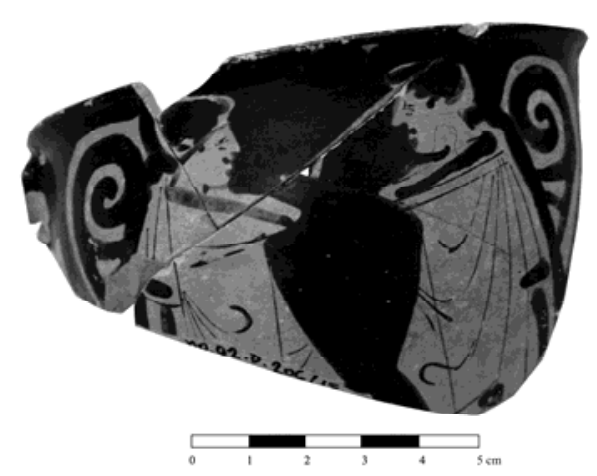

Fig. 6: Skyphos a figure rosse MD.02.206/9 (da Nigro 2004a, tav. XCIV).

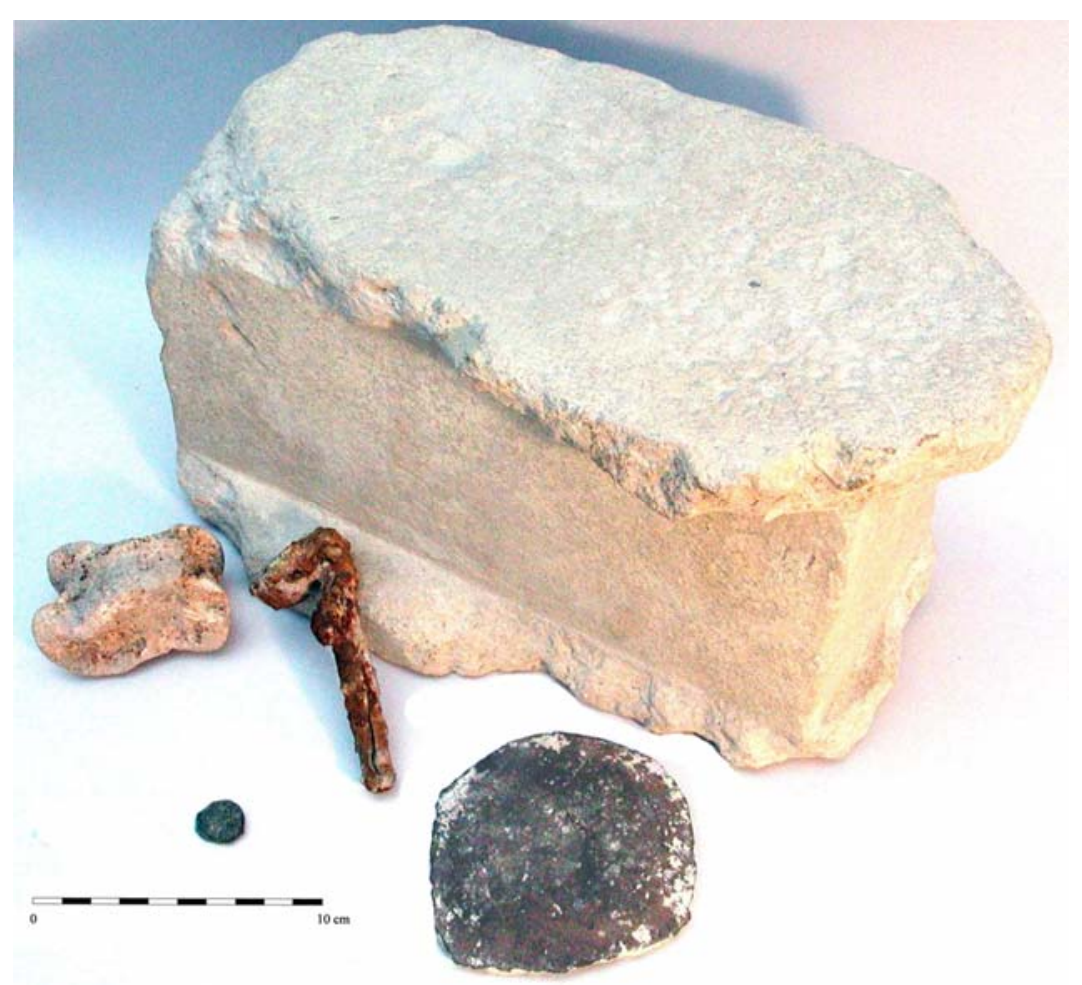

Fig. 7: Oggetti cultuali e votivi dal sacello domestico L.300 (da Nigro 2004a, tav. LI). 


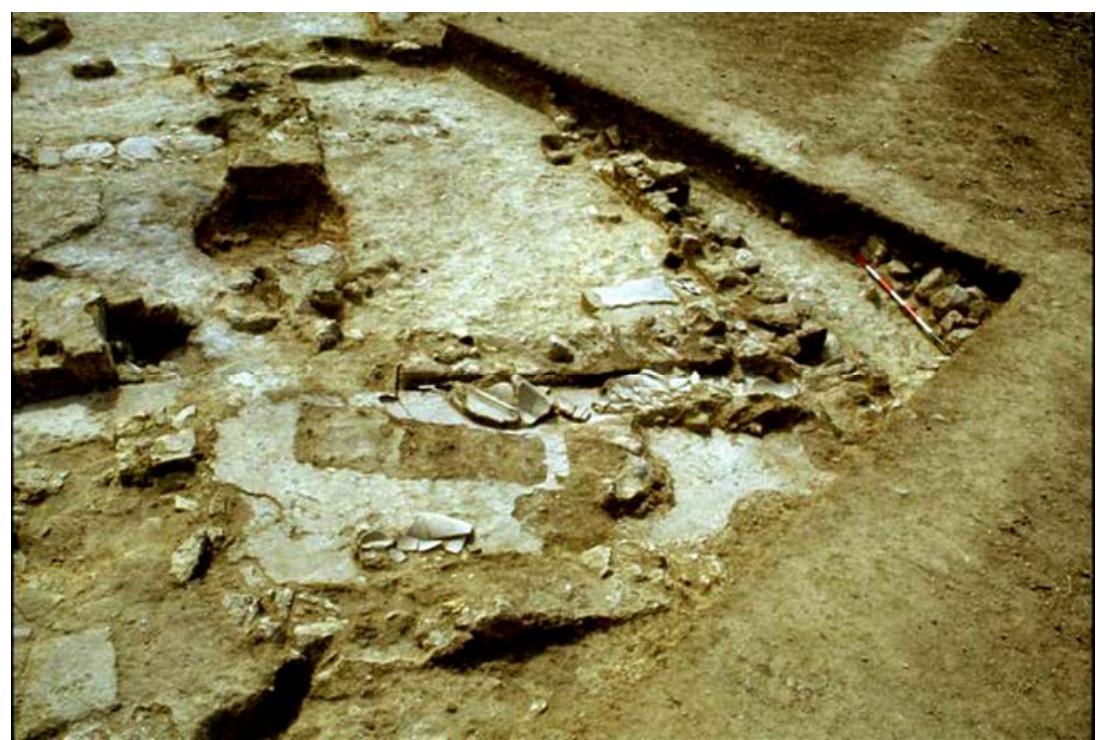

Fig. 8: "Casa del sacello domestico”, da nord; sulla sinistra, il sacello domestico L.300; in primo piano il bagno L.264; sul pavimento, frammenti di anfora e l'orlo della vasca a sabot.

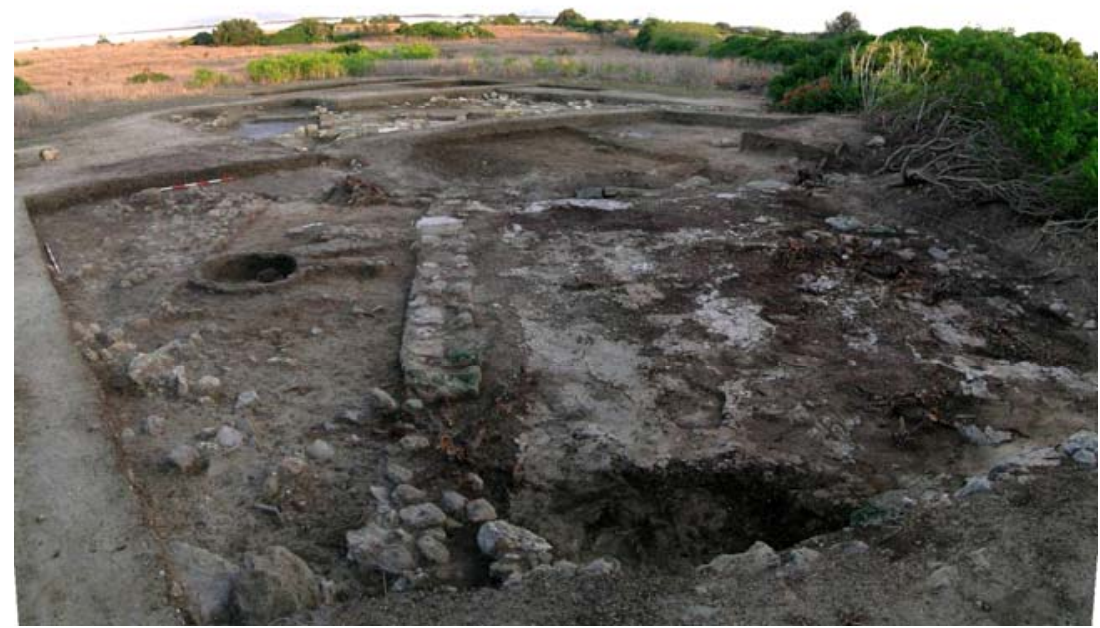

Fig. 9: “Casa del sacello domestico”, da est; sala da pranzo e cucina con forno. 


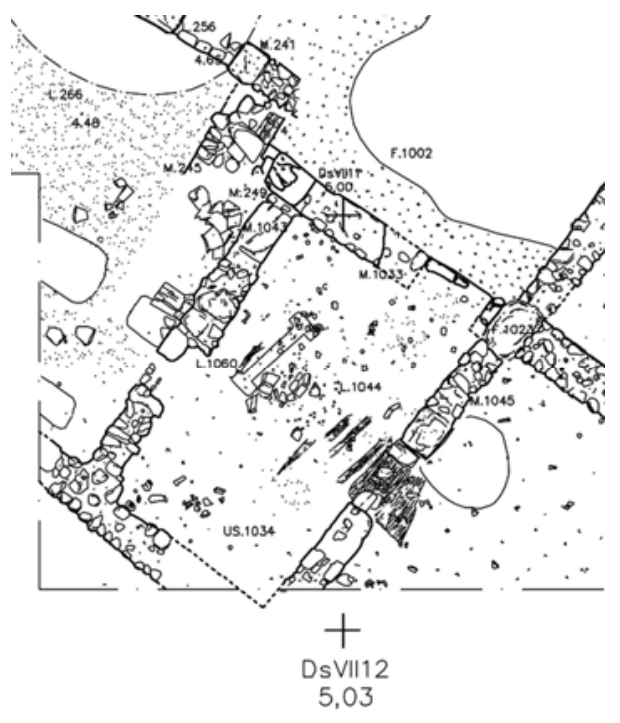

Fig. 10: "Casa del sacello domestico"; pianta dell'ambiente L.1044.

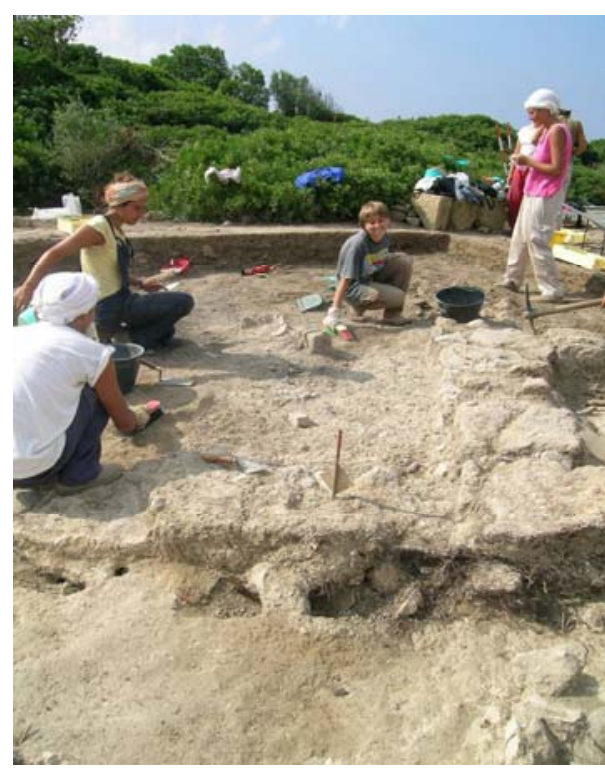

Fig. 11: Il rinvenimento dell'altare bruciaprofumi durante lo scavo del crollo in L.1044.

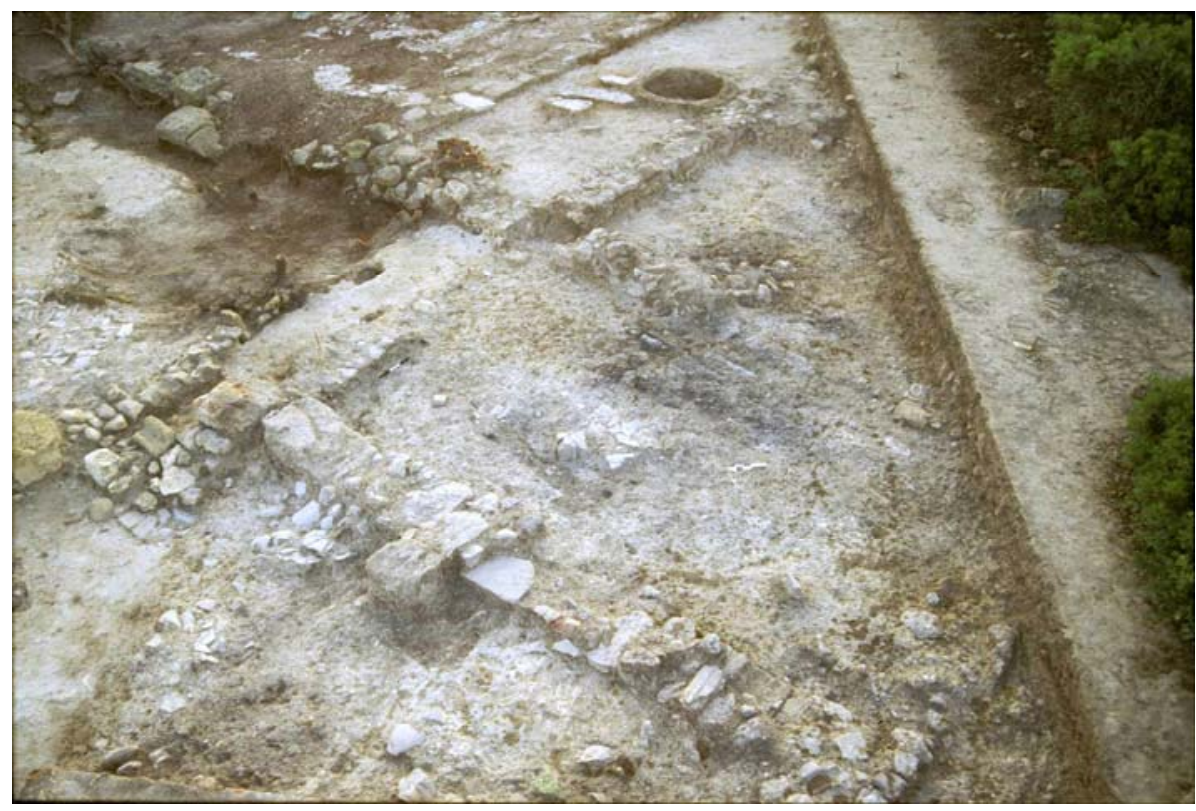

Fig. 12: L’impronta del telaio sul pavimento dell'ambiente L.1044, da sud ovest. 


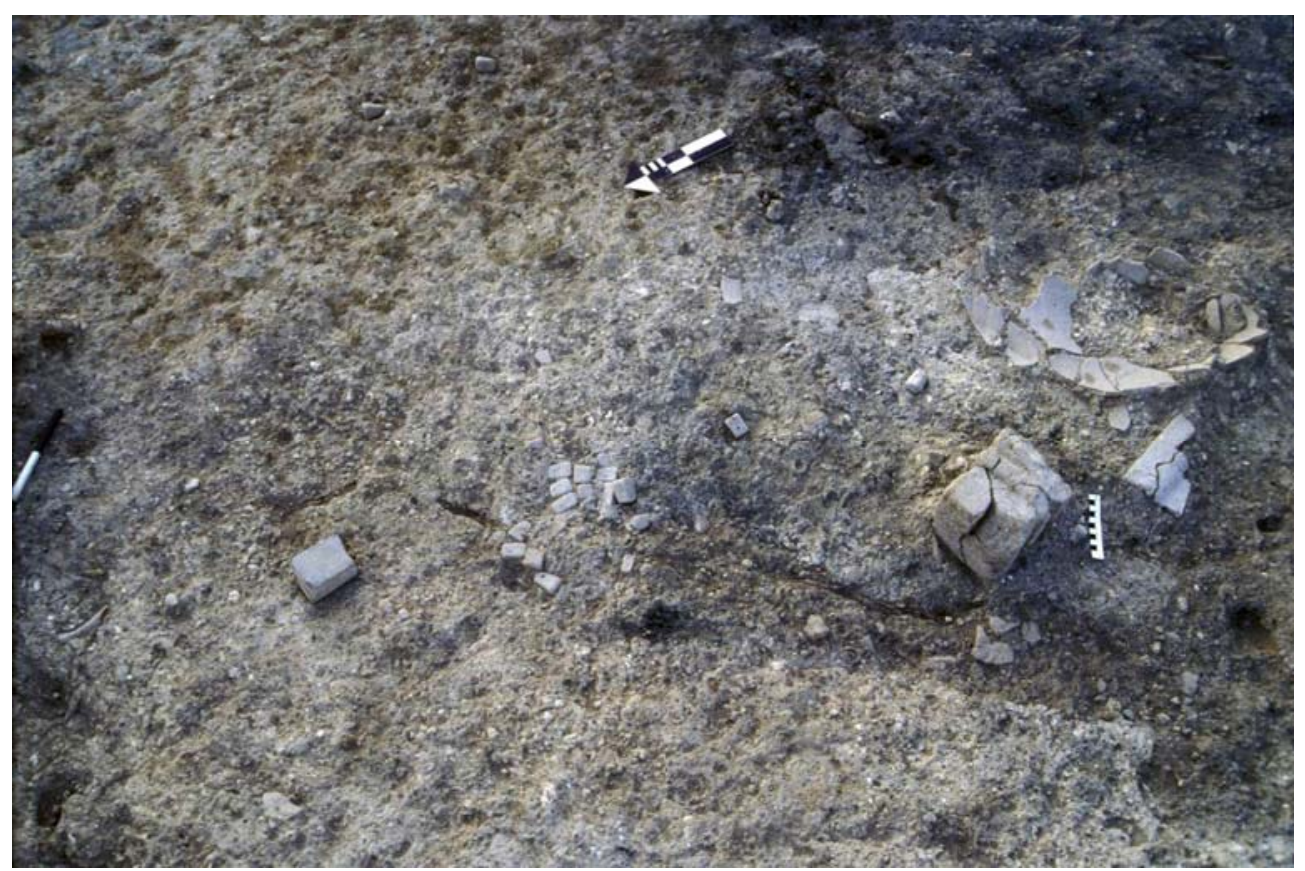

Fig. 13: Anfora punica e pesi da telaio contenuti originariamente in essa, rinvenuti schiacciati sul pavimento del vano L.1044.

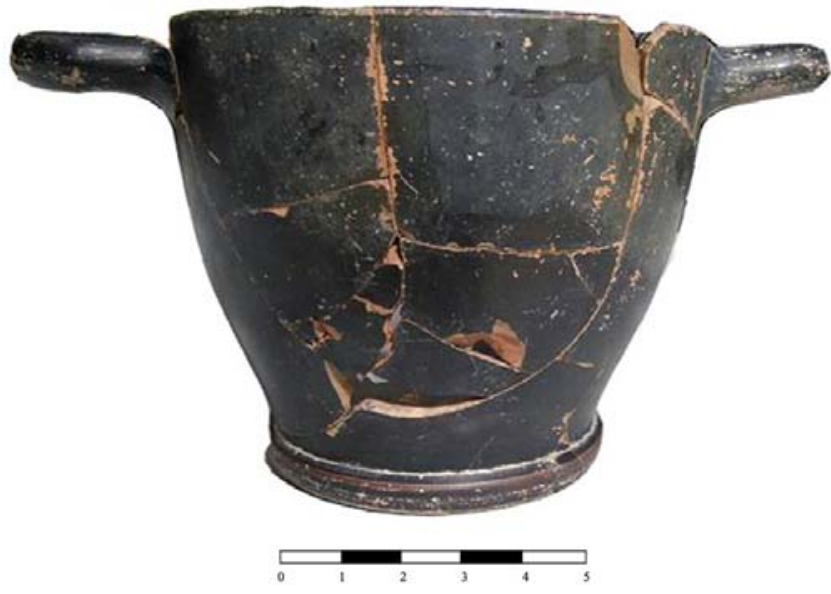

Fig. 14: Lo skyphos attico in vernice nera MD.03.1034/1 rinvenuto in frammenti in L.1044 (da Nigro 2007a, 196, tav. LXII). 
Fig. 15: L'altare bruciaprofumi (cat n. 1) MD.03.330 (da Nigro 2007a, 138, tav. XIV).
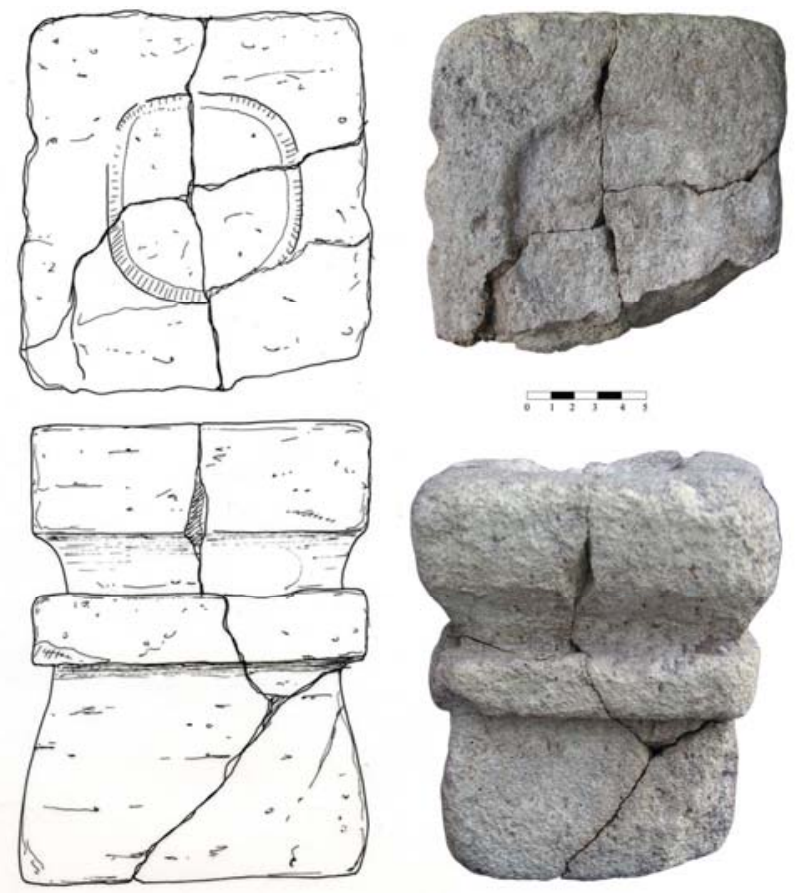

Fig. 16: Base-sostegno (cat. n. 2) rinvenuta nel tofet di Mozia (da Moscati - Uberti 1981, tav. CLXXXVI).
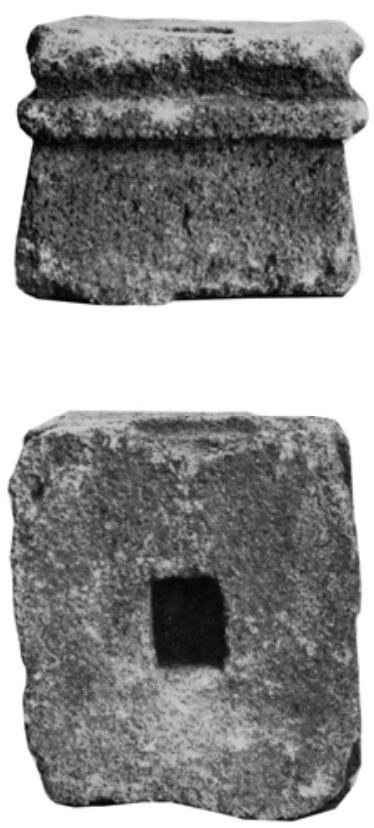


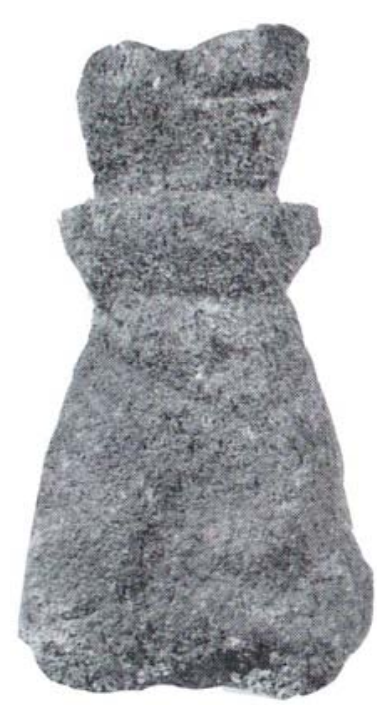

Fig. 17: Altare bruciaprofumi (cat. n. 3) dalla tomba T.63, necropoli di Palermo (da Tamburello 1967, fig. 21:a).

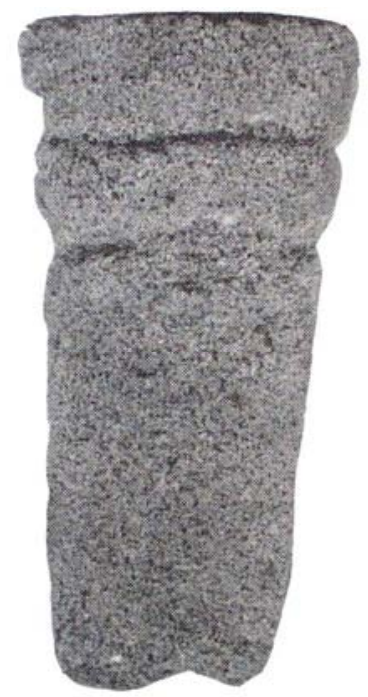

Fig. 19: Altare bruciaprofumi (cat. n. 5) dalla tomba T.269, necropoli di Palermo (da Tamburello 1967, fig. 21:b).

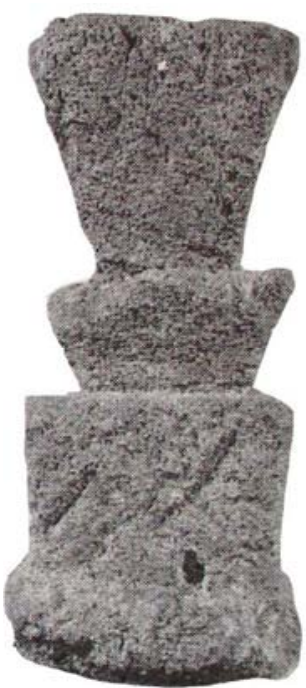

Fig. 18: Altare bruciaprofumi (cat. n. 4) dalla tomba T.185, necropoli di Palermo (da Tamburello 1967, fig. 21:c).

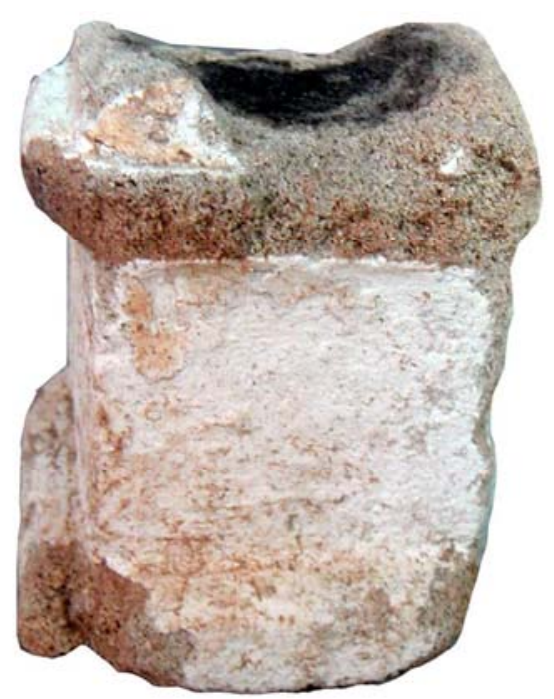

Fig. 20: Altarino bruciaprofumi (cat. n. 6) dall'abitato ellenistico di Solunto (foto autore). 


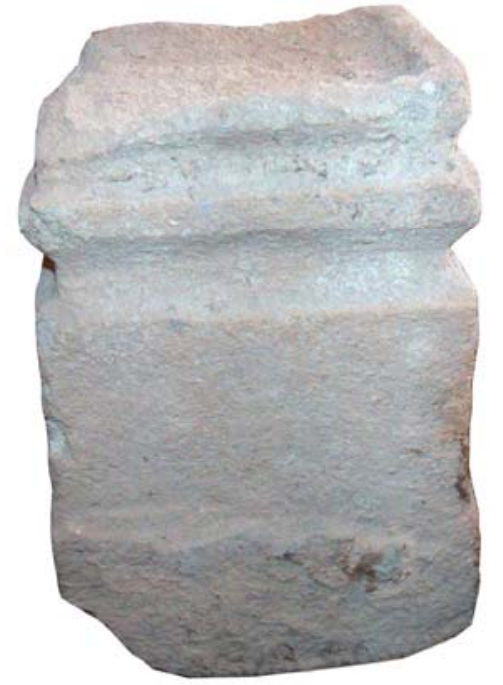

Fig. 21: Altare bruciaprofumi dalla necropoli punica di Solunto (foto autore).

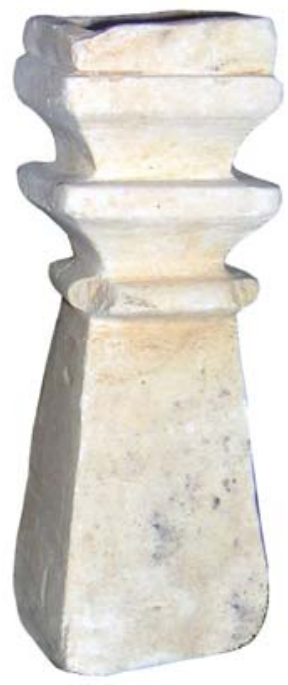

Fig. 23: Altare bruciaprofumi (cat. n. 8) dalla tomba L.13, necropoli di Byrsa, Cartagine (foto autore).

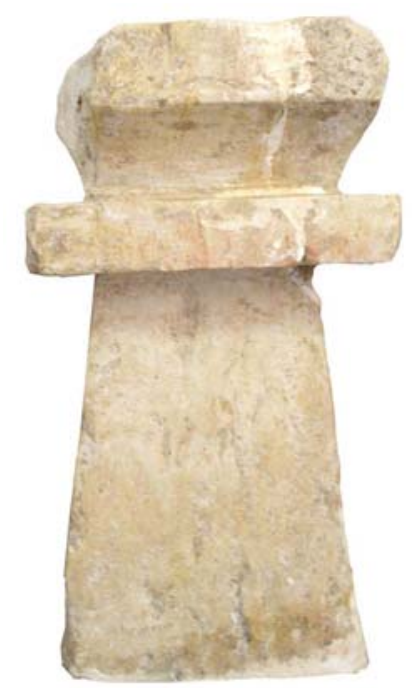

Fig. 22: “Altare di tipo egizio” (cat. n. 7) dal Santuario di Kerkouane (da Fantar 1986, tav. XCVI).

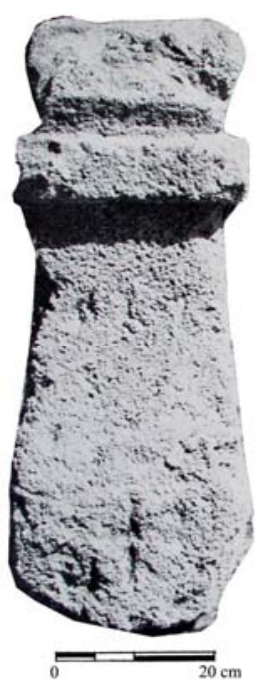

Fig. 24: Altare bruciaprofumi (cat. n. 9) dal tofet di Cartagine (da Bartoloni 1976, n. 43 , tav. XII). 

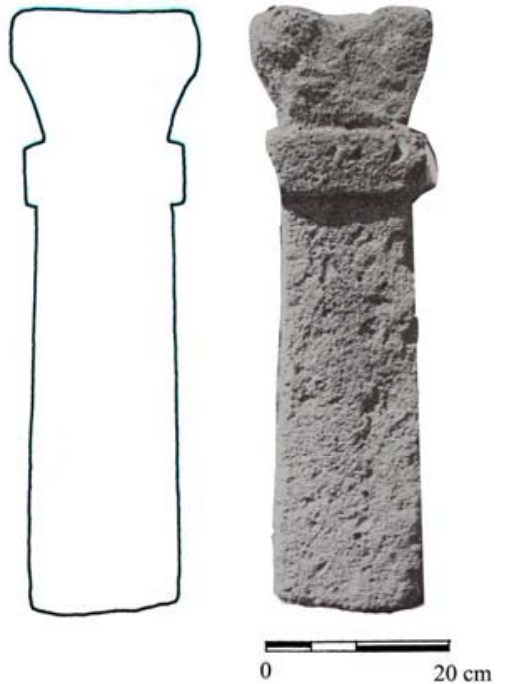

Fig. 25: Altare bruciaprofumi (cat. n. 10) dal tofet di Cartagine (da Bartoloni 1976, n. 44, tav. XII).

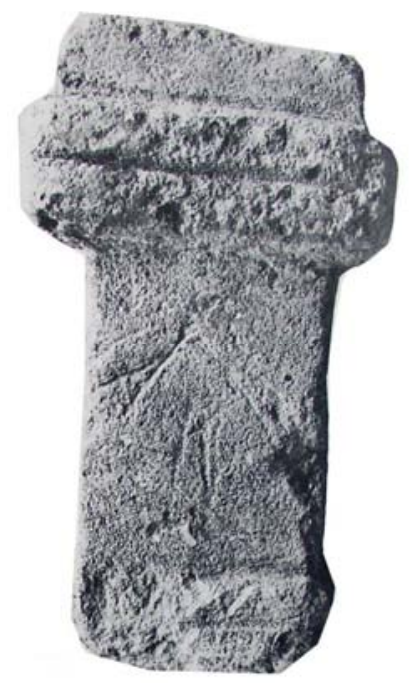

Fig. 27: Altare bruciaprofumi (cat. n. 12) dal Tempio di Tanit, Nora, (da Tore 1973, tav. XIX:2).

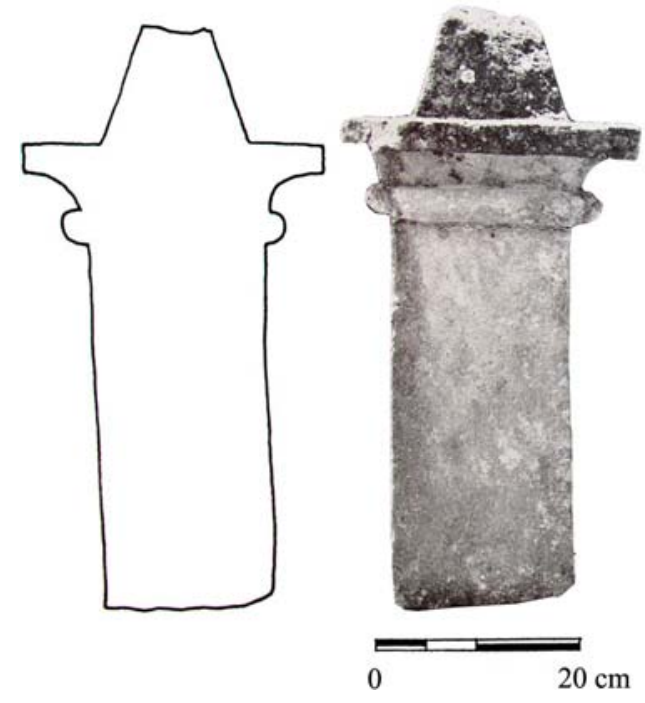

Fig. 26: Altare bruciaprofumi (cat. n. 11) dal tofet di Cartagine (da Bartoloni 1976, n. 46 , tav. XII).

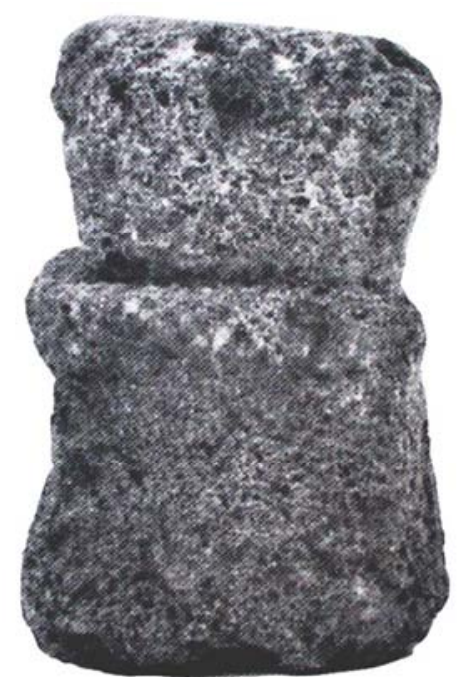

Fig. 28: Altare bruciaprofumi (cat. n. 13) dal tofet di Tharros (da Moscati - Uberti 1985, tav. LXXI). 


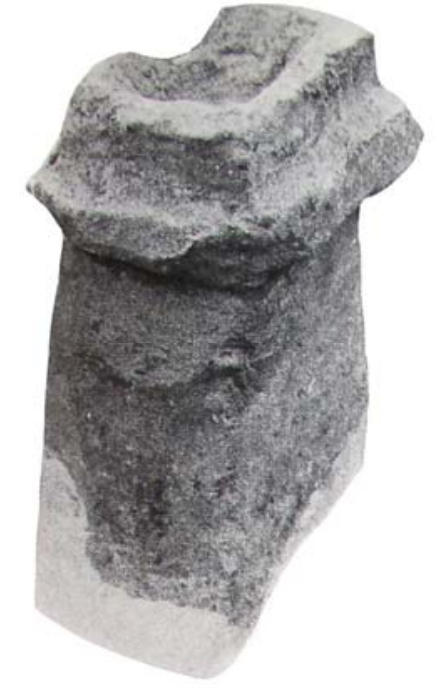

Fig. 29: Altare bruciaprofumi (cat. n. 14) dalla necropoli occidentale di Karalis (da Tore 1973, tav. XXIV:1).

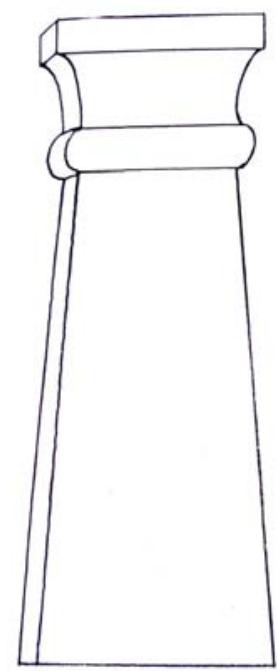

Fig. 31: Altare bruciaprofumi (cat. n. 16) dalla necropoli di Villaricos (da Astruc 1951, tav. L:1).

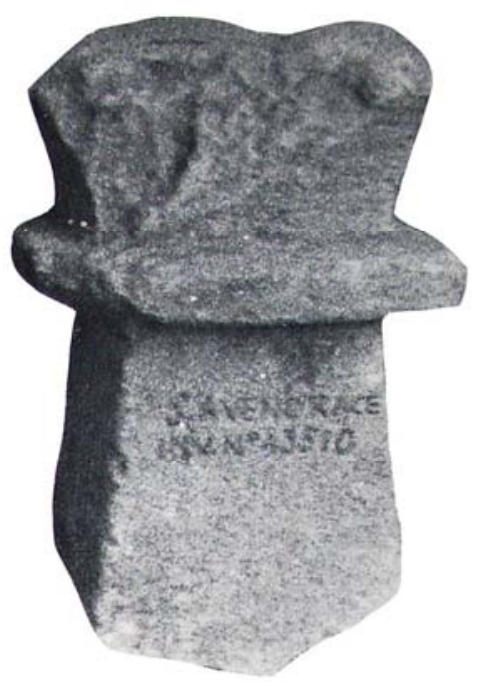

Fig. 30: Altare bruciaprofumi (cat. n. 15) dalla necropoli di Sant'Avendrace (da Tore 1973, tav. XXIV:2).

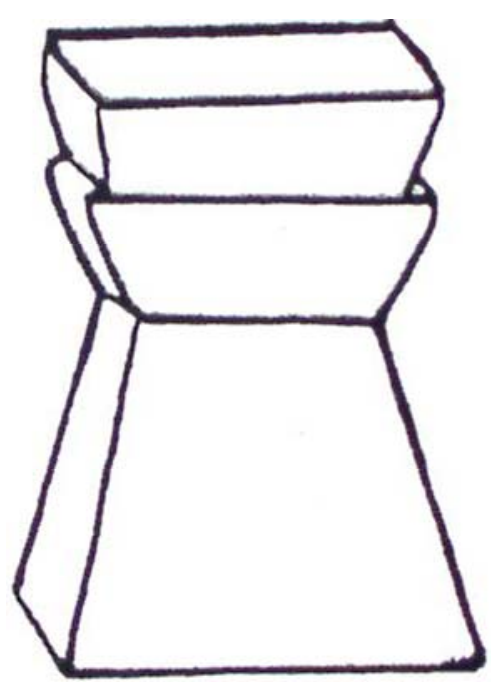

Fig. 32: Altare bruciaprofumi (cat. n. 17) dalla necropoli di Villaricos (da Astruc 1951, tav. L:4). 


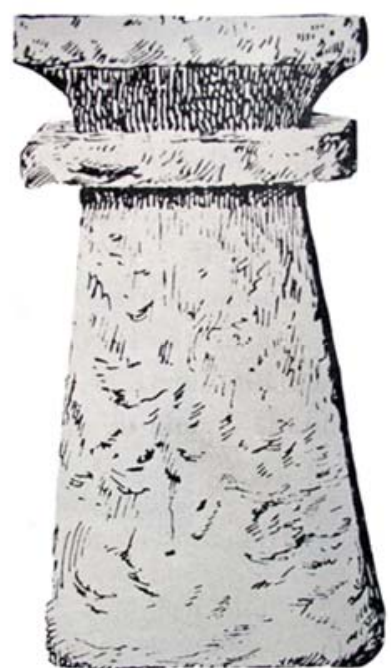

Fig. 33: Altare bruciaprofumi (cat. n. 18) da Huelva (da García y Bellido 1942, fig. 57).

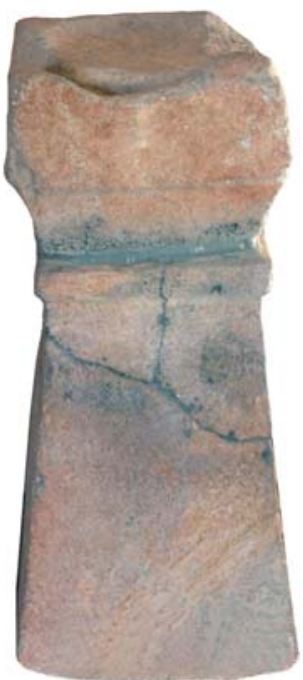

Fig. 34: Altarino bruciaprofumi dal Santuario di Afrodite, da KoukliaPalaepaphos (foto autore).

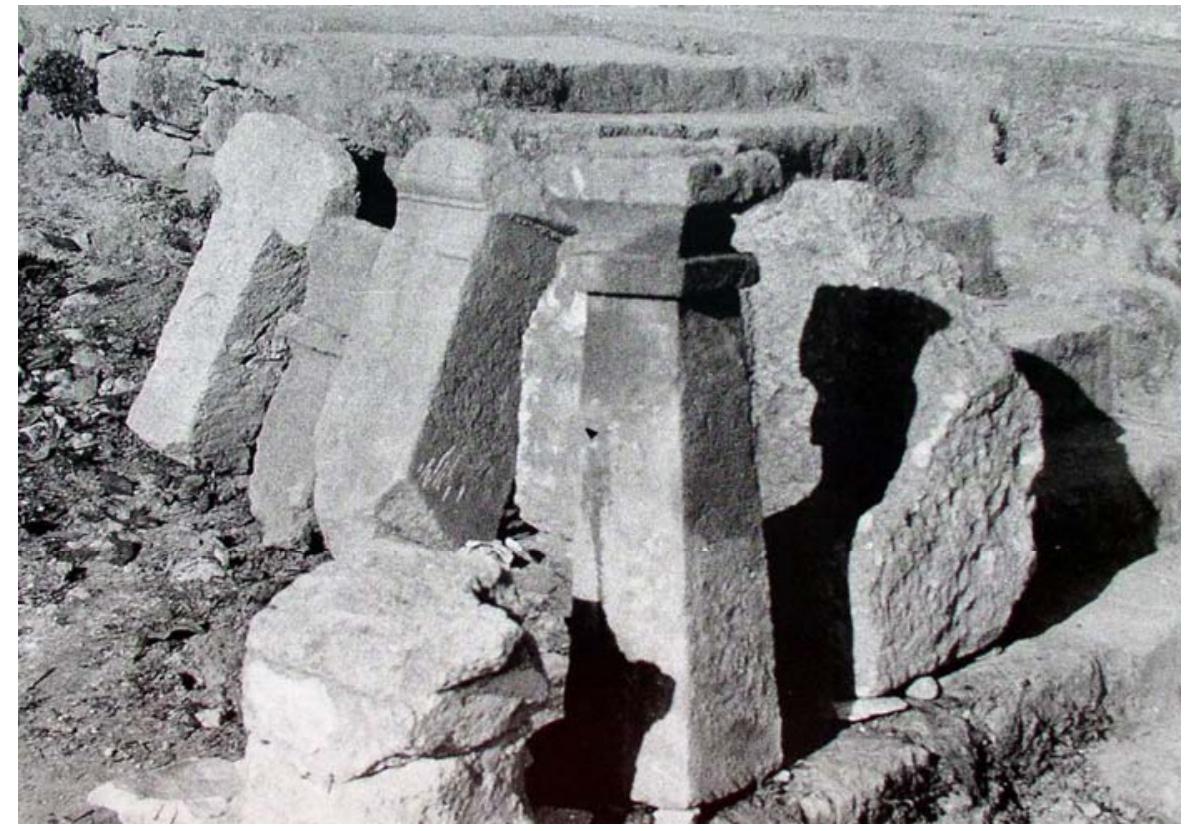

Fig. 35: Altari bruciaprofumi (cat. nn. 19-21) da Bostan es-Sheikh, Sidone (da Stucky 1993, tav. 16). 